\title{
Wild kernels at the prime 2
}

\author{
by \\ Paul Arne Østver (Oslo)
}

\section{Background and summary of results}

Background. Let $F$ be a number field. If $\wp$ is a prime of $F$, let $F_{\wp}$ denote the completion of $F$ at $\wp$. By Matsumoto's theorem, the second K-group $\mathrm{K}_{2}(F)$ of $F$ is the quotient of $F^{\times} \otimes F^{\times}$by the subgroup generated by the elements $x \otimes(1-x), x \neq 0,1$. The Hilbert symbols induce a map $\mathrm{K}_{2}(F) \rightarrow$ $\bigoplus \mu\left(F_{\wp}\right)$, where the direct sum is taken over the finite and real infinite primes of $F$. Its kernel is the Hilbert kernel or the wild kernel of $F$. Nontrivial elements in the wild kernel $\mathrm{WK}_{2}(F)$ correspond to exotic symbols, that is, symbols which can be detected by global class field theory but not by local class field theory. Much of the interest in the wild kernel is motivated by connections with number theory, for example the Leopoldt and Gross conjectures.

Let $S_{p}$ denote the set of primes above the rational prime $(p)$ in $F$, and let $R_{F}$ be the ring of $p$-integers of $F$. It follows from the reciprocity uniqueness theorem of Moore (see $[\mathrm{CW}]$ and $[\mathrm{Mo}]$ ) that the $p$-primary part of $\mathrm{WK}_{2}(F)$ for $p$ an odd prime number fits in an exact sequence

$$
0 \rightarrow \mathrm{WK}_{2}(F)\{p\} \rightarrow \mathrm{K}_{2}\left(R_{F}\right)\{p\} \rightarrow \bigoplus_{S_{p}} \mu\left(F_{\wp}\right)\{p\} \rightarrow \mu(F)\{p\} \rightarrow 0 .
$$

There is a similar exact sequence for the two-primary wild kernel: An important difference is that in addition to the dyadic primes, the sum includes the real infinite primes of the number field.

The higher wild kernels of $F$ give a generalization of (1.1). Assume $p$ is an odd prime number. In [Ba2], Banaszak defined the higher wild kernels $\mathrm{WK}_{2 n}(F)\{p\}$. As for the classical wild kernel, there are close connections between the higher wild kernels and number theory. We refer to [Ba1], [Ba2],

2000 Mathematics Subject Classification: 11R23, 11R37, 11R42, 11R70, 19D50, $19 \mathrm{~F} 27$.

Key words and phrases: higher wild kernels, étale cohomology, zeta-functions, global class field theory, Gross kernel, Fitting ideals, Leopoldt's conjecture, higher rank formulas, 2-divisible elements in algebraic K-groups. 
[BG1] and [BG2] for detailed discussions. In [B-Z], the Quillen-Lichtenbaum conjecture for $\mathbb{Q}$ at $p$ is shown to be equivalent to the statement that $\mathrm{WK}_{2 n}(\mathbb{Q})\{p\}$ is isomorphic to the group of $p$-divisible elements of $\mathrm{K}_{2 n}(\mathbb{Q})$. By [B-Z, Theorem $\mathrm{C}]$ and the Quillen-Lichtenbaum conjecture for local fields [HM], this also holds for arbitrary number fields. A proof of the BlochKato conjecture would imply the Quillen-Lichtenbaum conjecture for number fields at odd primes.

In this paper we consider two-primary higher wild kernels. Many of our results are inspired by the earlier results for $\mathrm{K}_{2}$ and the classical wild kernel. At the prime two, the real embeddings of a number field clutter up arguments which are valid for totally imaginary number fields, or more restrictively for non-exceptional number fields. An extension of our results for non-exceptional number fields to exceptional number fields would be of interest.

Summary of results. Let $n$ be an even natural number. In Section 4 we define the higher wild kernels $\mathrm{WK}_{n}(F)\{2\}$ of $F$ at the prime 2 . These groups are related to the algebraic K-groups of $F$, and to values of zeta-functions if $F$ is a totally real Abelian number field. If $A$ is an Abelian group, let ${ }_{n} A$ denote the subgroup of elements of exponent $n$. Let $e_{\nu}$ be the exponent of $\left(\mathbb{Z} / 2^{\nu}\right)^{\times}$. In Proposition 4.8 we show that ${ }_{2} \mathrm{WK}_{2 m e_{\nu}-2}(F)$ is independent of $m$ if $F$ is totally imaginary.

In Section 5 we give a class field theoretic description of the higher wild kernels. This part is elevated from work of Kolster [Ko1] on $\mathrm{K}_{2}$, and presumes that the number field $F$ is non-exceptional. The argument uses Iwasawa theory and Galois descent for the étale cohomology groups of the intermediate fields in the tower $\ldots \subset F\left(\mu_{2^{\nu}}\right) \subset F\left(\mu_{2^{\nu+1}}\right) \subset \ldots$

In Theorem 6.1 we show an isomorphism between the higher wild kernels and Galois coinvariants of Picard groups. This result generalizes Theorem 6.6 of $[\mathrm{Ke}]$.

If $F$ is a totally real Abelian extension of the rationals and $n \equiv 2 \bmod 4$, the main result in $[\mathrm{C} \varnothing]$ allows us to compute the Fitting ideals of eigenspaces of $\mathrm{WK}_{n}(F)\{2\}$. As conjectured by Coates and Sinnott [CS], each of these ideals are generated by a Stickelberger element. This result explicates elements which annihilate eigenspaces of the wild kernel. See Theorem 7.1 for the precise statement.

Assume $F$ has no real embeddings. We show that if $\mathrm{rk}_{2^{\nu}} \mathrm{WK}_{2 e_{\nu}-2}(F)$ $=0$, then Leopoldt's conjecture holds for $F$ at the prime 2. In Theorem 8.6 we obtain higher 2-rank formulas for the wild kernels. This part requires $F$ to be non-exceptional.

In the last section we discuss the group of divisible elements in $\mathrm{K}_{2 n}(F)$. If $F$ is a non-exceptional field, we note that $\mathrm{WK}_{2 n}(F)\{2\}$ is isomorphic 
to the group of 2-divisible elements in $\mathrm{K}_{2 n}(F)$. This result goes back to the works of Banaszak [Ba2] and Schneider [Sc]. It is an open problem to identify the 2-divisible elements for exceptional number fields. In [Hut], Hutchinson obtained a complete solution of this problem for the Hilbert kernel and any number field.

Finally, we point out that we do not define a wild kernel $\mathrm{WK}_{n}(F)\{2\}$ for $F$ a real number field and $n \equiv 4 \bmod 8$. This is quite unsatisfactory, but in general this case requires a lengthy discussion beyond the scope of this paper.

Acknowledgments. I thank Manfred Kolster for useful discussions. In particular, Section 5 and the rank formulas in Theorem 8.8 are inspired by Kolster's work on the classical wild kernel. We use the work of Keune [Ke] to prove Theorem 6.1. The results in Section 7 were established in collaboration with Pietro Cornacchia. I am also very grateful for the valuable comments of Cornelius Greither, Kevin Hutchinson, John Rognes, and a referee.

2. Tate-Poitou duality. Let $F$ be a number field. Denote by $R_{F}$ the ring of $p$-integers in $F$, and by $S_{p, \infty}$ the union of the $p$-adic primes $S_{p}$ and the Archimedean primes $S_{\infty}$ in $F$. Consider the étale cohomology groups $\mathrm{H}_{\text {ét }}^{n}\left(R_{F} ; M\right)$ for $M$ equal to $\mathbb{Z} / p^{\nu}(i), \mathbb{Z}_{p}(i)$ (see $[\mathrm{Ja}]$ ), or $\mathbb{Q}_{p} / \mathbb{Z}_{p}(i)$. If $p=2$, see $[\mathrm{C}-\mathrm{S}]$ for the definition of the positive étale cohomology groups $\mathrm{H}_{+}^{n}\left(R_{F} ; M\right)$. In our indexing, the positive group is trivial for $n \neq 1,2$. For notational convenience we let $\mathbb{Z} / p^{\nu}(i)^{\prime}$ denote $\mathbb{Z} / p^{\nu}(1-i)$, and likewise for $\mathbb{Z}_{p}(i)$ and $\mathbb{Q}_{p} / \mathbb{Z}_{p}(i)$. If $A$ is an Abelian group, let $A^{\#}$ denote its Pontryagin dual. In the following, let $\widehat{\mathrm{H}}$ denote Tate cohomology and let $S$ be a subset of $S_{p, \infty}$.

Definition 2.1. Let $\amalg_{S}^{n}\left(R_{F} ; M\right)$ denote the kernel of the localization map:

$$
\beta^{n}(M): \mathrm{H}_{\text {ét }}^{n}\left(R_{F} ; M\right) \rightarrow \bigoplus_{S} \mathrm{H}_{\text {ét }}^{n}\left(F_{\wp} ; M\right) .
$$

Let us recall Tate-Poitou duality (cf. [Mi] and [Ta1]):

TheOREM 2.2. There exists a natural 9-term exact sequence:

$$
\begin{aligned}
& 0 \longrightarrow \mathrm{H}_{\text {ét }}^{0}(F ; M) \stackrel{\beta^{0}(M)}{\longrightarrow} \bigoplus_{S_{p, \infty}} \widehat{\mathrm{H}}_{\mathrm{ét}}^{0}\left(F_{\wp} ; M\right) \stackrel{\gamma^{0}(M)}{\longrightarrow} \mathrm{H}_{\text {ét }}^{2}\left(R_{F} ; M^{\prime}\right)^{\#} \\
& \mathrm{H}_{\text {ét }}^{1}\left(R_{F} ; M^{\prime}\right)^{\#} \stackrel{\gamma^{1}(M)}{\longleftarrow} \bigoplus_{S_{p, \infty}} \mathrm{H}_{\text {ét }}^{1}\left(F_{\wp} ; M\right) \stackrel{\beta^{1}(M)}{\longleftarrow} \mathrm{H}_{\text {ét }}^{1}\left(R_{F} ; M\right)
\end{aligned}
$$

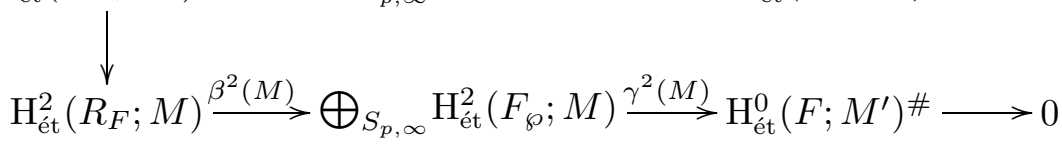


and perfect pairings:

$$
\begin{gathered}
\mathrm{H}_{\text {ét }}^{n}\left(F_{\wp} ; M\right) \times \mathrm{H}_{\text {ét }}^{2-n}\left(F_{\wp} ; M^{\prime}\right) \rightarrow \mathbb{Q} / \mathbb{Z}, \\
\amalg_{S}^{n}\left(R_{F} ; M\right) \times \amalg_{S}^{3-n}\left(R_{F} ; M^{\prime}\right) \rightarrow \mathbb{Q} / \mathbb{Z} .
\end{gathered}
$$

REMARK 2.3. If $p$ is odd, there is an isomorphism between the $p$-primary étale wild kernel $\mathrm{WK}_{2 i-2}^{\text {ét }}(F)\{p\}$ of $F$ and $\amalg_{S_{p}}^{2}\left(R_{F} ; \mathbb{Z}_{p}(i)\right)$ (cf. [Ko3] and [Ng2]).

3. Algebraic K-theory of number rings. In [RW], Rognes and Weibel used Voevodsky's proof of the Milnor conjecture to compute the two-primary algebraic K-groups of number rings. More precisely, see Theorems 0.4 and 0.6 in [RW] for the following result.

TheOREM 3.1. Let $n \geq 1$.

(a) If $F$ is totally imaginary, then $\mathrm{K}_{2 n}\left(R_{F}\right)\{2\}=\mathrm{H}_{\text {ét }}^{2}\left(R_{F} ; \mathbb{Z}_{2}(n+1)\right)$.

(b) If $F$ is a real number field, then

$$
\mathrm{K}_{2 n}\left(R_{F}\right)\{2\}= \begin{cases}\mathrm{H}_{\text {ét }}^{2}\left(R_{F} ; \mathbb{Z}_{2}(n+1)\right) & \text { if } n \equiv 0,1 \bmod 4, \\ \mathrm{H}_{+}^{2}\left(R_{F} ; \mathbb{Z}_{2}(n+1)\right) & \text { if } n \equiv 3 \bmod 4 .\end{cases}
$$

Let $\zeta_{F}$ denote the zeta-function of $F$, and let $r_{1}(F)$ denote the number of real embeddings of $F$. We write $a \sim_{2} b$ if $a$ and $b$ have the same 2-adic valuation. See [RW, Theorem 0.2] for the following important result:

TheOREM 3.2. Let $F$ be a totally real Abelian number field. Then for all even $n>0$,

$$
\zeta_{F}(1-n) \sim_{2} 2^{r_{1}(F)} \frac{\# \mathrm{~K}_{2 n-2}\left(R_{F}\right)\{2\}}{\# \mathrm{~K}_{2 n-1}\left(R_{F}\right)\{2\}} .
$$

We will employ Theorem 3.1 to define the two-primary higher wild kernels of $F$. If $F$ is a totally real Abelian number field, then Theorem 3.2 implies a formula for the order of these groups.

4. Higher wild kernels. In this section we introduce the higher wild kernels at the prime 2. The label (a) refers to totally imaginary number fields, and (b) refers to real number fields. The positive étale cohomology groups in Theorem 3.1 fit into the exact sequences $\bigoplus^{r_{1}} \mathrm{H}_{\text {êt }}^{1}\left(\mathbb{R} ; \mathbb{Z}_{2}(i)\right) \rightarrow$ $\mathrm{H}_{+}^{2}\left(R_{F} ; \mathbb{Z}_{2}(i)\right) \rightarrow \mathrm{H}_{\text {ét }}^{2}\left(R_{F} ; \mathbb{Z}_{2}(i)\right) \rightarrow \bigoplus^{r_{1}} \mathrm{H}_{\text {êt }}^{2}\left(\mathbb{R} ; \mathbb{Z}_{2}(i)\right) \rightarrow 0$. The group $\mathrm{H}_{\text {ét }}^{2}\left(\mathbb{R} ; \mathbb{Z}_{2}(i)\right)$ is (1) isomorphic to $\mathbb{Z} / 2$ if $i$ is even, and (2) trivial if $i$ is odd. If $i$ is even, then $\mathrm{H}_{\text {êt }}^{1}\left(\mathbb{R} ; \mathbb{Z}_{2}(i)\right)$ is the trivial group. In particular, $\mathrm{H}_{+}^{2}\left(R_{F} ; \mathbb{Z}_{2}(i)\right)$ injects into $\mathrm{H}_{\text {ét }}^{2}\left(R_{F} ; \mathbb{Z}_{2}(i)\right)$. It follows that Theorems 2.2 and 3.1 imply: 
Corollary 4.1. Let $n \geq 1$.

(a) There is an exact sequence

$$
\mathrm{K}_{2 n}\left(R_{F}\right)\{2\} \stackrel{\beta_{n}^{2}}{\longrightarrow} \bigoplus_{S_{2}} \mathrm{H}_{\text {ét }}^{2}\left(F_{\wp} ; \mathbb{Z}_{2}(n+1)\right) \rightarrow \mathrm{H}_{\text {ét }}^{0}\left(F ; \mathbb{Q}_{2} / \mathbb{Z}_{2}(n)\right) \rightarrow 0 .
$$

(b) Assume $n \not \equiv 2 \bmod 4$. Then there are exact sequences

$$
\mathrm{K}_{2 n}\left(R_{F}\right)\{2\} \stackrel{\beta_{n}^{2}}{\longrightarrow} \bigoplus_{S} \mathrm{H}_{\text {ét }}^{2}\left(F_{\wp} ; \mathbb{Z}_{2}(n+1)\right) \rightarrow \mathrm{H}_{\text {ét }}^{0}\left(F ; \mathbb{Q}_{2} / \mathbb{Z}_{2}(n)\right) \rightarrow 0,
$$

where $S=S_{2, \infty}$ if $n \equiv 1 \bmod 4$ and $S=S_{2}$ otherwise.

The kernel of $\beta_{1}^{2}$ is the two-primary part of the classical wild kernel of $F$. This fact motivates the next definition.

Definition 4.2. The $n$th two-primary wild kernel $\mathrm{WK}_{2 n}(F)\{2\}$ of $F$ is the kernel of the map $\beta_{n}^{2}$ in Corollary 4.1.

Next we compute the index of the higher wild kernels in the higher tame kernels. If $k$ is field and $i \in \mathbb{Z}$, we let $w_{i}^{(2)}(k)$ be the maximal power $2^{n}$ of 2 such that the exponent of $\operatorname{Gal}\left(k\left(\zeta_{2^{n}}\right) / k\right)$ divides $i$. This number equals $\# \mathrm{H}_{\text {ét }}^{0}\left(k ; \mathbb{Q}_{2} / \mathbb{Z}_{2}(i)\right)$.

In the next result, the case of $\mathrm{K}_{2}$ is due to Tate (see [CL, Proposition 5.1]).

Proposition 4.3. We have the following calculations:

(a)

$$
\frac{\# \mathrm{~K}_{2 n}\left(R_{F}\right)\{2\}}{\# \mathrm{WK}_{2 n}(F)\{2\}}=\frac{\prod_{S_{2}} w_{n}^{(2)}\left(F_{\wp}\right)}{w_{n}^{(2)}(F)} ;
$$

$$
\begin{aligned}
\frac{\# \mathrm{~K}_{8 n}\left(R_{F}\right)\{2\}}{\# \mathrm{WK}_{8 n}(F)\{2\}} & =\frac{\prod_{S_{2}} w_{4 n}^{(2)}\left(F_{\wp}\right)}{w_{4 n}^{(2)}(F)}, \\
\frac{\# \mathrm{~K}_{8 n+2}\left(R_{F}\right)\{2\}}{\# \mathrm{WK}_{8 n+2}(F)\{2\}} & =2^{r_{1}(F)-1} \prod_{S_{2}} w_{4 n+1}^{(2)}\left(F_{\wp}\right), \\
\frac{\# \mathrm{~K}_{8 n+6}\left(R_{F}\right)\{2\}}{\# \mathrm{WK}_{8 n+6}(F)\{2\}} & =\frac{1}{2} \prod_{S_{2}} w_{4 n+3}^{(2)}\left(F_{\wp}\right) .
\end{aligned}
$$

Proof. Consider the case (b) and $\mathrm{WK}_{8 n+2}(F)$. By Corollary 4.1 we have $\# \mathrm{~K}_{8 n+2}\left(R_{F}\right)\{2\} \cdot \# \mathrm{H}_{\text {ét }}^{0}\left(F ; \mathbb{Q}_{2} / \mathbb{Z}_{2}(4 n+1)\right)$

$$
=2^{r_{1}} \cdot \# \mathrm{WK}_{8 n+2}(F)\{2\} \cdot \# \bigoplus_{S_{2}} \mathrm{H}_{\text {ét }}^{0}\left(F_{\wp} ; \mathbb{Q}_{2} / \mathbb{Z}_{2}(4 n+1)\right) .
$$

If $i$ is odd, then $w_{i}^{(2)}(F)=2$ since $F$ is a real number field. The other cases follow similarly. 
For totally real Abelian number fields, the cardinalities of the twoprimary higher wild kernels are determined by the values at odd negative integers of the associated zeta-functions:

Proposition 4.4. Let $F$ be a totally real Abelian number field. Then

$$
\begin{gathered}
\# \mathrm{WK}_{8 n+2}(F)\{2\} \sim_{2} \frac{\zeta_{F}(-4 n-1) w_{4 n+2}^{(2)}(F)}{2^{r_{1}(F)-1} \prod_{S_{2}} w_{4 n+1}^{(2)}\left(F_{\wp}\right)}, \\
\# \mathrm{WK}_{8 n+6}(F)\{2\} \sim_{2} \frac{\zeta_{F}(-4 n-3) w_{4 n+4}^{(2)}(F)}{2^{r_{1}(F)-1} \prod_{S_{2}} w_{4 n+3}^{(2)}\left(F_{\wp}\right)} .
\end{gathered}
$$

Proof. See [RW, Theorem 6.14(b)], Theorem 3.2 and Proposition 4.3.

A number field $F$ is called two-regular if the 2-Sylow subgroup of its modified tame kernel is trivial [GJ]. An equivalent condition is that (2) does not split in $\mathcal{O}_{F}$ and the narrow Picard group $\operatorname{Pic}_{+}\left(R_{F}\right)$ has odd order.

Lemma 4.5. Let $F$ be a two-regular number field. Then $\mathrm{WK}_{2 n}(F)\{2\}$ is the trivial group.

Proof. Theorems 3.1 and 4.1 in [RØ] show that $\mathrm{K}_{2 n}\left(R_{F}\right)\{2\}=0$ unless $r_{1}(F)>0$ and $n \equiv 1 \bmod 4$. It remains to show that $\amalg_{S_{2, \infty}}^{2}\left(R_{F} ; \mathbb{Z}_{2}(4 n+2)\right)$ $=0$ if $n \geq 0$. By Theorem 2.2 it suffices to prove that $\gamma^{0}\left(\mathbb{Q}_{2} / \mathbb{Z}_{2}(-4 n-1)\right)$ is a surjective map. We have $\mathrm{H}_{\text {ét }}^{2}\left(R_{F} ; \mathbb{Z}_{2}(4 n+2)\right)=(\mathbb{Z} / 2)^{r_{1}}$ by $[\mathrm{R} \varnothing$, Proposition 2.2]. Note that the group $\widehat{\mathrm{H}}_{\text {ét }}^{0}\left(\mathbb{C} ; \mathbb{Q}_{2} / \mathbb{Z}_{2}(-4 n-1)\right)$ is trivial, but $\widehat{\mathrm{H}}_{\text {ét }}^{0}\left(\mathbb{R} ; \mathbb{Q}_{2} / \mathbb{Z}_{2}(-4 n-1)\right)=\mathbb{Z} / 2$. Hence $\gamma^{0}\left(\mathbb{Q}_{2} / \mathbb{Z}_{2}(-4 n-1)\right)$ is a map from an $\mathbb{F}_{2}$-vector space of dimension $r_{1}+1$ to an $\mathbb{F}_{2}$-vector space of dimension $r_{1}$. Its kernel is cyclic of order 2 by Theorem 2.2 (cf. the proof of Proposition 4.3). The map in question is therefore surjective.

See $[\mathrm{Hu}]$ for a list of all real quadratic number fields whose wild kernel is trivial. Proposition 4.4 and Lemma 4.5 give the 2 -adic valuation of $\zeta_{F}(-n)$ for $n \geq 1$ an odd integer and $F$ a totally real two-regular Abelian number field. We write $t$ for the 2-rank of $\operatorname{Pic}\left(R_{F}\right)$, and $u$ for the 2-rank of $\operatorname{Pic}_{+}\left(R_{F}\right)$.

Lemma 4.6. Let $F$ be a number field. In case (b) and $n \equiv 3 \bmod 4$, we have $\mathrm{rk}_{2} \mathrm{WK}_{2 n}(F) \geq u-1$. In all other cases we have $\mathrm{rk}_{2} \mathrm{WK}_{2 n}(F) \geq t-1$.

If $F$ is a totally real number field, then $\amalg_{S_{2, \infty}}^{1}\left(R_{F} ; \mathbb{Q}_{2} / \mathbb{Z}_{2}(4 n+2)\right)$ injects into $\mathrm{WK}_{8 n+2}(F)\{2\}$ and $\amalg_{S_{2}}^{1}\left(R_{F} ; \mathbb{Q}_{2} / \mathbb{Z}_{2}(4 n+4)\right)$ injects into $\mathrm{WK}_{8 n+6}(F)\{2\}$.

Proof. The 2-rank of $\mathrm{K}_{n}\left(R_{F}\right)$ is computed in [RW, Theorem 7.11]. By comparing with Corollary 4.1, the lower bounds for $\mathrm{rk}_{2} \mathrm{WK}_{2 n}(F)$ follow.

Let $M=\mathbb{Q}_{2} / \mathbb{Z}_{2}(4 n+2)$ and $N=\mathbb{Z}_{2}(4 n+2)$. If $F$ is a totally real number field, the Bockstein map $\beta: \mathrm{H}_{\text {ét }}^{1}\left(R_{F} ; M\right) \rightarrow \mathrm{H}_{\text {ét }}^{2}\left(R_{F} ; N\right)$ in the 
universal coefficient sequence is an isomorphism (cf. [RW, Proposition 6.11]). We conclude using the following diagram with exact rows:

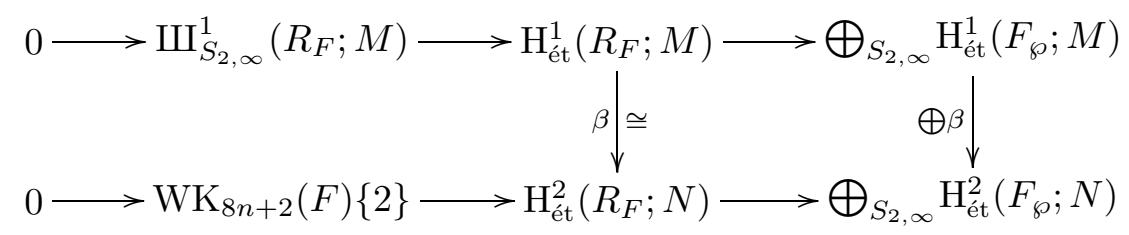

The same argument applies to $\mathrm{WK}_{8 n+6}\left(R_{F}\right)$.

Lemma 4.7. Assume $F$ contains a primitive $2^{\nu}$ th root of unity. If $\nu=1$ and $F$ is real, assume $n \equiv 0,1 \bmod 4$. Let $S$ be as in Corollary 4.1. If $\amalg_{S}^{2}\left(R_{F} ; \mathbb{Z}_{2}(n+1)\right)=\amalg_{S}^{2}\left(R_{F} ; \mathbb{Z} / 2^{\nu}(n+1)\right)$, then $\mathrm{WK}_{2 n}(F)\{2\}$ is isomorphic to $\left(\operatorname{Pic}\left(R_{F}\right) / 2^{\nu}\right)(n)$.

Proof. Duality and the assumption $\mu_{2^{\nu}} \in F$ imply the isomorphisms

$$
\amalg_{S}^{2}\left(R_{F} ; \mathbb{Z} / 2^{\nu}(n+1)\right)=\amalg_{S}^{1}\left(R_{F} ; \mathbb{Z} / 2^{\nu}(-n)\right)^{\#}=\amalg_{S}^{1}\left(R_{F} ; \mathbb{Z} / 2^{\nu}\right)^{\#}(n) .
$$

On the other hand, by class field theory we have

$$
\amalg_{S}^{1}\left(R_{F} ; \mathbb{Z} / 2^{\nu}\right)=\operatorname{Hom}\left(\operatorname{Pic}\left(R_{F}\right), \mathbb{Z} / 2^{\nu}\right)=\left(\operatorname{Pic}\left(R_{F}\right) / 2^{\nu}\right)^{\#} .
$$

Proposition 4.8. Assume $F$ is totally imaginary, and let $e_{\nu}$ denote the exponent of $\left(\mathbb{Z} / 2^{\nu}\right)^{\times}$. Let $m$ be any natural number such that $m e_{\nu}>1$. Then the groups ${ }_{2}{ }^{\nu} \mathrm{K}_{2 m e_{\nu}-2}\left(R_{F}\right)$ are independent of $m$. Likewise for ${ }_{2}{ }^{2} \mathrm{WK}_{2 m e_{\nu}-2}\left(R_{F}\right)$.

Proof. By [RW, Theorem 6.14(a)], the two-torsion in $\mathrm{K}_{2 n-1}(F)$ is cyclic of order $w_{n}^{(2)}(F)$ for $n \geq 2$. Hence ${ }_{2} \mathrm{~K}_{2 n-1}(F)=\mathrm{H}_{\text {ét }}^{0}\left(F ; \mathbb{Z} / 2^{\nu}(n)\right)$. The absolute Galois group of $F$ acts trivially on the $m e_{\nu}$-fold tensor product of the group of $2^{\nu}$ th roots of unity, so $\mathrm{K}_{2 m e_{\nu}-1}(F)$ contains an element of order $2^{\nu}$ and ${ }_{2^{\nu}} \mathrm{K}_{2 n-1}(F)={ }_{2^{\nu}} \mathrm{K}_{2\left(n+m e_{\nu}\right)-1}(F)$. The proof of [RW, Theorem 7.2] shows that $\mathrm{K}_{2 m e_{\nu}-1}\left(R_{F} ; \mathbb{Z} / 2^{\nu}\right)$ is isomorphic to $\mathrm{H}_{\text {ét }}^{1}\left(R_{F} ; \mathbb{Z} / 2^{\nu}\left(m e_{\nu}\right)\right)$ for all $\nu \geq 1$. By the Bockstein exact sequence in $\bmod 2^{\nu}$-homotopy, there is the exact sequence

$$
0 \rightarrow \mathrm{K}_{2 m e_{\nu}-1}(F) / 2^{\nu} \rightarrow \mathrm{H}_{\text {ét }}^{1}\left(R_{F} ; \mathbb{Z} / 2^{\nu}\left(m e_{\nu}\right)\right) \rightarrow{ }^{\nu} \mathrm{K}_{2 m e_{\nu}-2}\left(R_{F}\right) \rightarrow 0 .
$$

This sequence is split since $\mathrm{K}_{2 m e_{\nu}-1}(F) / 2^{\nu}=\left(\mathbb{Z} / 2^{\nu}\right)^{r_{2}+1}$ is a pure subgroup of $\mathrm{H}_{\text {ét }}^{1}\left(R_{F} ; \mathbb{Z} / 2^{\nu}\left(m e_{\nu}\right)\right)$. Two of the groups in the split exact sequence are therefore independent of $m$, and so is the third by the cancellation property of finite groups.

Consider now the wild kernels. In [Ko3, pp. 48-49] a map

$$
\alpha / 2^{\nu}\left(R_{F}, m e_{\nu}\right): \mathrm{H}_{\text {ét }}^{1}\left(R_{F} ; \mathbb{Z} / 2^{\nu}\left(m e_{\nu}\right)\right) \rightarrow \bigoplus_{S_{2}} 2^{\nu} \mathrm{H}_{\text {ét }}^{2}\left(F_{\wp} ; \mathbb{Z}_{2}\left(m e_{\nu}\right)\right)
$$


is constructed with kernel independent of $m$ (cf. Section 5). The source of this map surjects onto ${ }_{2} \mathrm{~K}_{2 m e_{\nu}-2}\left(R_{F}\right)$ by the exact sequence above, while its target receives a map from the same group by Corollary 4.1(a). The kernel-cokernel exact sequence of the resulting diagram is

$$
0 \rightarrow \mathrm{K}_{2 m e_{\nu}-1}(F) / 2^{\nu} \rightarrow \operatorname{ker} \alpha / 2^{\nu}\left(R_{F}, m e_{\nu}\right) \rightarrow{ }_{2} \mathrm{WK}_{2 m e_{\nu}-2}\left(R_{F}\right) \rightarrow 0,
$$

and we may proceed as above.

5. An idélic description of the wild kernels. In this section we will relate the higher wild kernels to idéles in class field theory. Our approach is inspired by Kolster's [Ko1] idélic description of the classical wild kernel.

Let $F_{k}=F\left(\zeta_{2^{k}}\right)$ and let $F_{\infty}=\bigcup_{k} F_{k}$. Each $F_{k}$ is a Galois extension of $F$, and $F$ is called non-exceptional if $\Gamma^{\prime}=\operatorname{Gal}\left(F_{\infty} / F\right)$ is isomorphic to the additive group of the two-adic integers. This occurs precisely if $\zeta_{4} \in F$, or $\zeta_{2^{k}}-\bar{\zeta}_{2^{k}} \in F$ for some $k \geq 3$. Let $R_{F_{k}}$ denote the ring of two-integers in $F_{k}$, and let $R_{F_{\infty}}$ be the union. The roots of unity contained in $F_{k}$ allow us to use the constructions of [Ko1]. If $F$ is non-exceptional, we use Galois descent arguments to conclude for $F$.

Let $\beta(i)$ be the Bockstein map, and consider the commutative diagram:

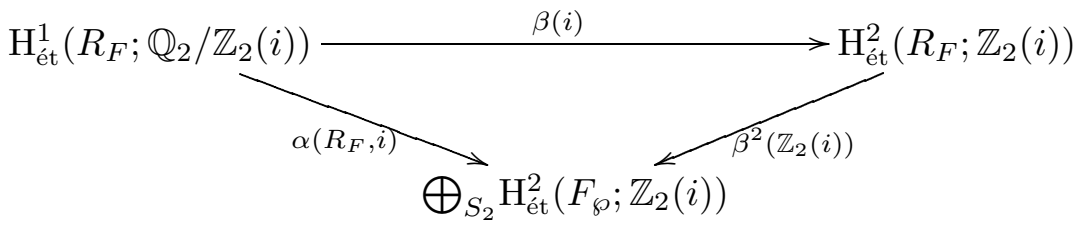

The kernel-cokernel sequence of (5.1) is

$$
0 \rightarrow\left(\mathbb{Q}_{2} / \mathbb{Z}_{2}\right)^{r(F)} \rightarrow \operatorname{ker} \alpha\left(R_{F}, i\right) \rightarrow \amalg_{S_{2}}^{2}\left(R_{F} ; \mathbb{Z}_{2}(i)\right) \rightarrow 0,
$$

where $r(F)=r_{2}(F)$ if $i \geq 2$ is even, and $r(F)=r_{1}(F)+r_{2}(F)$ if $i \geq 3$ is odd. An alternative description of $\operatorname{ker} \alpha\left(R_{F}, i\right)$ is as follows: Let $\nu_{\wp}$ be the normalized discrete valuation at a prime $\wp$, and put

$$
\Delta_{F}^{\nu}=\left\{1 \otimes u \in \mathbb{Z} / 2^{\nu} \otimes F^{\times} \mid \nu_{\wp}(u) \equiv 0 \bmod 2^{\nu} \text { for all } \wp \notin S_{2, \infty}\right\} .
$$

As $\nu$ varies, there are compatible isomorphisms

$$
\Delta_{F}^{\nu} \stackrel{\cong}{\longrightarrow} \mathrm{H}_{\text {ét }}^{1}\left(R_{F} ; \mathbb{Z} / 2^{\nu}(1)\right) \text {. }
$$

If $1 \leq \nu \leq k$, the cup-product map with a generator of $\mathrm{H}_{\text {ét }}^{0}\left(R_{F_{k}} ; \mathbb{Z} / 2^{\nu}(i-1)\right)$ induces isomorphisms by (5.4):

$$
\Delta_{F_{k}}^{\nu}(i-1) \stackrel{\cong}{\longrightarrow} \mathrm{H}_{\text {ét }}^{1}\left(R_{F_{k}} ; \mathbb{Z} / 2^{\nu}(i)\right) .
$$

Let $\alpha / 2^{\nu}\left(R_{F}, i\right)$ denote the map $\alpha\left(R_{F}, i\right)$ pre-composed with the natural map from $\mathrm{H}_{\text {ét }}^{1}\left(R_{F} ; \mathbb{Z} / 2^{\nu}(i)\right)$ to $\mathrm{H}_{\text {ét }}^{1}\left(R_{F} ; \mathbb{Q}_{2} / \mathbb{Z}_{2}(i)\right)$. 
Definition 5.6. Define the $(i-1)$ th twisted Kummer radical $D_{F_{k}}^{\nu}(i-1)$ for $1 \leq \nu \leq k$ to be the pre-image of $\operatorname{ker} \alpha / 2^{\nu}\left(R_{F_{k}}, i\right)$ under the isomorphism (5.5).

Consider the classical Kummer radical of $F$ :

$$
\begin{aligned}
D_{F}^{\nu}=\left\{\zeta_{2^{\nu}} \otimes u \in \mu_{2^{\nu}} \otimes F^{\times} \mid u=a_{\wp} b_{\wp}^{2^{\nu}}\right. & \text { for all } \wp \\
& \text { with } \left.a_{\wp} \in \mathcal{N}_{\wp} \text { and } b_{\wp} \in F_{\wp}^{\times}\right\} .
\end{aligned}
$$

Here $\mathcal{N}_{\wp}=\bigcap_{m>0} N\left(F_{\wp}\left(\zeta_{2^{m}}\right)^{\times}\right)$is the subgroup of $F_{\wp}^{\times}$of norms from the cyclotomic $\mathbb{Z}_{2}$-extension of $F_{\wp}$. In fact, $D_{F_{k}}^{\nu}(1)$ is the classical Kummer radical of $F_{k}$. To see this, consider the diagram:

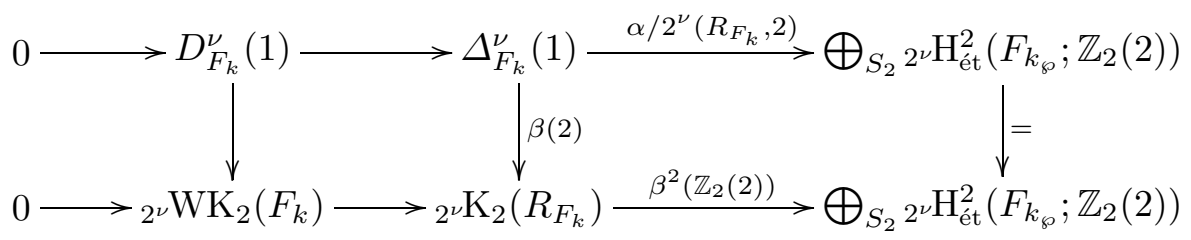

The right hand square commutes by definition of $\alpha / 2^{\nu}\left(R_{F_{k}}, 2\right)$, so there is a unique map $D_{F_{k}}^{\nu}(1) \rightarrow{ }_{2}^{\nu} \mathrm{WK}_{2}\left(F_{k}\right)$ that makes the left square commutative. The lower rightmost map is induced by the Hilbert symbol $(\cdot, \cdot)_{\wp}$ associated to $\wp$. Theorem 6.1 of [Ta3] shows that every element in ${ }_{2} \mathrm{~K}_{2}\left(F_{k}\right)$ can be written in the form $\left\{\zeta_{2^{\nu}}, u\right\}$, where $u$ is a unit in $F_{k}$. In particular, we have

$$
D_{F_{k}}^{\nu}(1)=\left\{\zeta_{2^{\nu}} \otimes u \in \mu_{2^{\nu}} \otimes F_{k}^{\times} \mid\left(\zeta_{2^{\nu}}, u\right)_{\wp}=1 \text { for all finite } \wp\right\} .
$$

Assume $\zeta_{2^{e}} \in F_{\wp}$, but $\zeta_{2^{e+1}} \notin F_{\wp}$. Recall that the Hilbert symbol $\left(\zeta_{2^{\nu}}, u\right)_{\wp}$ is trivial if and only if $u$ is a norm of the extension $F_{\wp}\left(\zeta_{2^{\nu}+e}\right) / F_{\wp}$. Furthermore, $N\left(F_{\wp}\left(\zeta_{2^{e+\nu}}\right)^{\times}\right)=\mathcal{N}_{\wp} F_{\wp}^{\times 2^{\nu}}$. By local class field theory, it follows that the left hand side of the equation equals $\bigcap_{m \geq \nu} F_{\wp}^{\times 2^{\nu}} N\left(F_{\wp}\left(\zeta_{2^{e+m}}\right)^{\times}\right)$since $F_{\wp}\left(\zeta_{2^{e+\nu}}\right)=F_{\wp}\left(\sqrt[2^{\nu}]{F_{\wp}^{\times}}\right) \cap F_{\wp}\left(\zeta_{2^{e+m}}\right)$ for $m \geq \nu$. It is clear that $\mathcal{N}_{\wp} F_{\wp}^{\times 2^{\nu}}$ is contained in the above intersection. The reverse inclusion holds by the example of [BP, p. 526]. See also [Gi, Lemma 1].

There are natural maps $D_{F_{k}}^{\nu}(i-1) \rightarrow D_{F_{k+1}}^{\nu}(i-1)$, and we denote the colimit by $D_{F_{\infty}}^{\nu}(i-1)$. Likewise we obtain $D_{F_{\infty}}^{\infty}(i-1)$. The cup-product and the direct limit functor are compatible, so the cup-product map induces an isomorphism

$$
D_{F_{\infty}}^{\nu}(i-1)=\operatorname{ker} \alpha / 2^{\nu}\left(R_{F_{\infty}}, i\right) .
$$

Next we consider Galois descent for $D_{F_{\infty}}^{\infty}(i-1)$.

Lemma 5.8. Assume $F$ is a non-exceptional number field, and $i \neq 0,1$. There is a Galois descent isomorphism $\operatorname{ker} \alpha\left(R_{F}, i\right)=D_{F_{\infty}}^{\infty}(i-1)^{\Gamma^{\prime}}$.

Proof. Consider the Lyndon-Hochschild-Serre spectral sequence

$$
E_{2}^{m, n}=\mathrm{H}^{m}\left(\Gamma^{\prime}, \mathrm{H}_{\text {ét }}^{n}\left(R_{F_{\infty}} ; \mathbb{Q}_{2} / \mathbb{Z}_{2}(i)\right)\right) \Rightarrow \mathrm{H}_{\text {ét }}^{m+n}\left(R_{F} ; \mathbb{Q}_{2} / \mathbb{Z}_{2}(i)\right) .
$$


The cohomological dimension of $\Gamma^{\prime}$ is 1 , so the following sequence is exact:

$$
0 \rightarrow E_{2}^{1,0} \rightarrow \mathrm{H}_{\text {ét }}^{1}\left(R_{F} ; \mathbb{Q}_{2} / \mathbb{Z}_{2}(i)\right) \rightarrow E_{2}^{0,1} \rightarrow 0 .
$$

Choose a topological generator $\gamma$ of $\Gamma^{\prime}$. Then $\mathrm{H}^{1}\left(\Gamma^{\prime} ; \mathbb{Q}_{2} / \mathbb{Z}_{2}(i)\right)$ is the cokernel of the map $1-\gamma: \mathbb{Q}_{2} / \mathbb{Z}_{2}(i) \rightarrow \mathbb{Q}_{2} / \mathbb{Z}_{2}(i)$, i.e., multiplication by $1-\kappa(\gamma)^{i}$ on $\mathbb{Q}_{2} / \mathbb{Z}_{2}$. Here $\kappa: \Gamma^{\prime} \rightarrow \mathbb{Z}_{2}^{\times}$denotes the cyclotomic character. It follows that $E_{2}^{1,0}$ is trivial if $i \neq 0$.

There is an isomorphism $\left(\bigoplus_{\bar{\wp} \mid \wp} \mathrm{H}_{\text {ét }}^{2}\left(F_{k_{\bar{\zeta}}} ; \mathbb{Z}_{2}(i)\right)\right)^{\Gamma_{k}}=\mathrm{H}_{\text {ét }}^{2}\left(F_{\wp} ; \mathbb{Z}_{2}(i)\right)$ for $\wp$ a dyadic prime and $i \neq 1$. This follows by local duality (cf. [KM, p. 44]).

Consider now, for $i \neq 0,1$, the diagram:

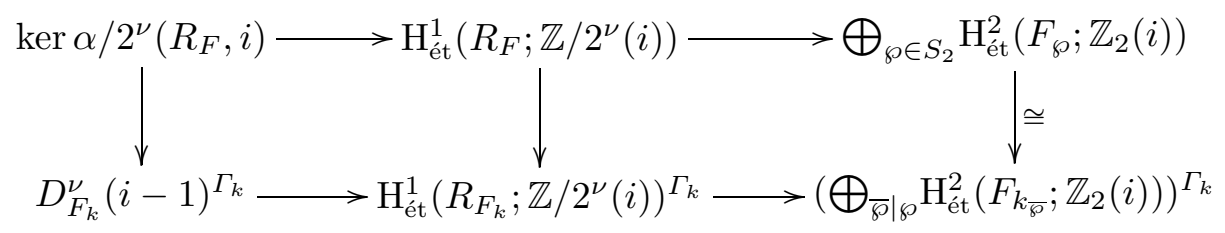

Here the left horizontal maps are injective. In the diagram obtained by taking the direct limit over $k$ and then over $\nu$, all vertical maps will be isomorphisms by the above.

The map $R_{F}^{\times} \rightarrow \bigoplus_{S_{p}} \mathbb{Z}_{p} \cdot \wp, u \mapsto \sum_{S_{p}} \log _{p}\left(N_{F_{\wp} / \mathbb{Q}_{p}}(u)\right) \cdot \wp$, extends by linearity to a map $g_{F}$ of $\mathbb{Z}_{p}$-modules. Its kernel is called the Gross kernel of $F$. We have the equality $\operatorname{rk}_{\mathbb{Z}_{p}}\left(\operatorname{ker} g_{F}\right)=r_{1}(F)+r_{2}(F)+\delta_{F}$ where $\delta_{F} \geq 0$ is the Gross defect. The following extension of the Gross conjecture [Gr] is due to Jaulent [Jau].

Conjecture 5.9. The Gross defect equals zero.

Conjecture 5.9 holds for Abelian number fields by [Jau] and [Gr]. Let $J_{F}$ denote the idéle group of $F$, and write $\mathbf{F}$ for the group of principal idéles. Define $\mathcal{N}_{\wp}$ to be $F_{\wp}^{\times}$for $\wp$ infinite, and let $X_{F}=\prod_{\wp} \mathcal{N}_{\wp}$. We use an overline to denote the closure in the idéle topology. The next result is Corollary 1.11 and Theorem 1.12 of [Ko1].

THEOREM 5.10. There is a split short exact sequence

$$
0 \rightarrow \operatorname{ker} g_{F} \otimes \mathbb{Q}_{2} / \mathbb{Z}_{2} \rightarrow D_{F}^{\infty} \rightarrow J_{F} / \overline{\mathbf{F} X_{F}}\{2\} \rightarrow 0 .
$$

By inserting $F_{k}$ for $F$ and passing to the colim over $k$, we obtain

$$
0 \rightarrow \operatorname{ker} g_{F_{\infty}} \otimes \mathbb{Q}_{2} / \mathbb{Z}_{2} \rightarrow D_{F_{\infty}}^{\infty} \rightarrow J_{F_{\infty}} / \overline{\mathbf{F}_{\infty} X_{F_{\infty}}}\{2\} \rightarrow 0 .
$$

Next we record a version of (5.11) for arbitrary twists.

Lemma 5.12. Assume $F$ is a non-exceptional number field. There is a short exact sequence 


$$
\begin{aligned}
0 \rightarrow\left(\operatorname{ker} g_{F_{\infty}} \otimes \mathbb{Q}_{2} / \mathbb{Z}_{2}(i-1)\right)^{\Gamma^{\prime}} & \rightarrow D_{F_{\infty}}^{\infty}(i-1)^{\Gamma^{\prime}} \\
& \rightarrow J_{F_{\infty}} / \overline{\mathbf{F}_{\infty} X_{F_{\infty}}}(i-1)\{2\}^{\Gamma^{\prime}} \rightarrow 0
\end{aligned}
$$

for $i \neq 1$, and for $i=1$ if $\delta_{F_{n}}=0$ for all $n$.

Proof. We must show that $\left(\operatorname{ker} g_{F_{\infty}} \otimes \mathbb{Q}_{2} / \mathbb{Z}_{2}(i-1)\right)_{\Gamma^{\prime}}$ is the trivial group. If $i \neq 1$, this follows from Tate's lemma [Ta2]. The case $i=1$ is implicit in [Ko1]. A proof can be given along the following lines: Let $\mathcal{X}_{\infty}$ be the Galois group of the maximal two-ramified Abelian pro-2-extension of $F_{\infty}$. Recall from [Iw, §7] the Kummer perfect pairing of $\Gamma^{\prime}$-modules:

$$
\mathcal{X}_{\infty}(-1) \times \Delta_{F_{\infty}}^{\infty} \rightarrow \mathbb{Q}_{2} / \mathbb{Z}_{2}
$$

Let $\mathcal{N}$ be the orthogonal complement of the $\Lambda$-torsion module tor ${ }_{\Lambda} \mathcal{X}_{\infty}(-1)$ of $\mathcal{X}_{\infty}(-1)$, and let $\mathrm{fr}_{\Lambda} \mathcal{X}_{\infty}(-1)$ be the quotient of $\mathcal{X}_{\infty}(-1)$ by tor $\mathcal{X}_{\infty}(-1)$. By [Ko1] we have $\mathcal{N}=\operatorname{ker} g_{F_{\infty}} \otimes \mathbb{Q}_{2} / \mathbb{Z}_{2}$ under the given hypothesis. The following sequence is therefore exact by (5.11):

$$
\begin{aligned}
0 \rightarrow \mathcal{N}^{\Gamma^{\prime}} \rightarrow\left(D_{F_{\infty}}^{\infty}\right) & \Gamma^{\Gamma^{\prime}} \rightarrow\left(J_{F_{\infty}} / \overline{\mathbf{F}_{\infty} X_{F_{\infty}}}\{2\}\right)^{\Gamma^{\prime}} \\
& \rightarrow \mathcal{N}_{\Gamma^{\prime}} \rightarrow\left(D_{F_{\infty}}^{\infty}\right)_{\Gamma^{\prime}} \rightarrow\left(J_{F_{\infty}} / \overline{\mathbf{F}_{\infty} X_{F_{\infty}}}\{2\}\right)_{\Gamma^{\prime}} \rightarrow 0 .
\end{aligned}
$$

From the pairing (5.14) it follows that $\mathcal{N}_{\Gamma^{\prime}}=\operatorname{Hom}_{\mathbb{Z}_{2}}\left(\operatorname{fr}_{\Lambda} \mathcal{X}_{\infty}(-1)^{\Gamma^{\prime}}, \mathbb{Q}_{2} / \mathbb{Z}_{2}\right)$ $=0$. Note that $\left(D_{F_{\infty}}^{\infty}\right)_{\Gamma^{\prime}}$ is finite.

Next we compare the sequences (5.2) and (5.13) assuming the hypothesis in Lemma 5.12 and $i \geq 2$. Lemma 5.8 gives an isomorphism between the two middle terms of these exact sequences, and hence $\left(\mathbb{Q}_{2} / \mathbb{Z}_{2}\right)^{r_{2}(F)}$ is the maximal divisible subgroup of $D_{F_{\infty}}^{\infty}(i-1)^{\Gamma^{\prime}}$. This group is contained in $\left(\operatorname{ker} g_{F_{\infty}} \otimes \mathbb{Q}_{2} / \mathbb{Z}_{2}(i-1)\right)^{\Gamma^{\prime}}$ by the finiteness of $J_{F_{\infty}} / \overline{\mathbf{F}_{\infty} X_{F_{\infty}}}(i-1)\{2\}^{\Gamma^{\prime}}$. By the snake lemma, we get the short exact sequence

$$
\begin{array}{r}
0 \rightarrow\left(\operatorname{ker} g_{F_{\infty}} \otimes \mathbb{Q}_{2} / \mathbb{Z}_{2}(i-1)\right)^{\Gamma^{\prime}} /\left(\mathbb{Q}_{2} / \mathbb{Z}_{2}\right)^{r_{2}(F)} \rightarrow \amalg_{S_{2}}^{2}\left(R_{F} ; \mathbb{Z}_{2}(i)\right) \\
\rightarrow J_{F_{\infty}} / \overline{\mathbf{F}_{\infty} X_{F_{\infty}}}(i-1)\{2\}^{\Gamma^{\prime}} \rightarrow 0 .
\end{array}
$$

TheOREM 5.16. Let $F$ be a non-exceptional number field and $n \geq 2$. Then there is an exact sequence

$$
\begin{aligned}
0 \rightarrow\left(\operatorname{ker} g_{F_{\infty}} \otimes \mathbb{Q}_{2} / \mathbb{Z}_{2}(n-1)\right)^{\Gamma^{\prime}} & \left(\mathbb{Q}_{2} / \mathbb{Z}_{2}\right)^{r_{2}(F)} \rightarrow \mathrm{WK}_{2 n-2}(F)\{2\} \\
& \rightarrow J_{F_{\infty}} / \overline{\mathbf{F}_{\infty} X_{F_{\infty}}}(n-1)\{2\}^{\Gamma^{\prime}} \rightarrow 0 .
\end{aligned}
$$

Proof. Use (5.15) and the definition of the wild kernel.

REMARK 5.17. For odd prime numbers, there is an analogous exact sequence for any number field. There is also a version at each finite level (cf. (8.5)). 
6. The Picard group description. The main result in this section is:

THEOREM 6.1. Let $F$ be a non-exceptional number field and $n \geq 1$. Choose $\nu$ such that $w_{n}^{(2)}\left(F_{\wp}\right) \leq 2^{\nu}$ for all dyadic primes $\wp$ in $F$, and such that $2^{\nu}$ annihilates the two-primary part of $\mathrm{K}_{2 n}\left(R_{F}\right)$. If $\Gamma=\operatorname{Gal}\left(F\left(\zeta_{2^{\nu}}\right) / F\right)$, there is an isomorphism

$$
\left(\operatorname{Pic}\left(R_{F\left(\zeta_{2^{\nu}}\right)}\right) / 2^{\nu}(n)\right)_{\Gamma}=\mathrm{WK}_{2 n}(F)\{2\} .
$$

In Theorem 6.1, the case $n=1$ is due to Keune (see [Ke, Theorem 6.6]). If $n \geq 2$, our proof follows the same line of argument. We will only discuss the intermediate steps in some details.

The field $F$ is totally imaginary, so the $n$th two-primary wild kernel of $F$ fits into the exact sequence

$$
\begin{aligned}
0 \rightarrow \mathrm{WK}_{2 n}(F)\{2\} & \rightarrow \mathrm{K}_{2 n}\left(R_{F}\right)\{2\} \\
& \rightarrow \bigoplus_{S_{2}} \mathrm{H}_{\text {ét }}^{2}\left(F_{\wp} ; \mathbb{Z}_{2}(n+1)\right) \rightarrow \mathrm{H}_{\text {ét }}^{0}\left(F ; \mathbb{Q}_{2} / \mathbb{Z}_{2}(n)\right) \rightarrow 0 .
\end{aligned}
$$

Concerning codescent for $\mathrm{K}_{2 n}\left(R_{F}\right)$ we have the following result:

Proposition 6.3. Let $n \geq 1$. Assume $E / F$ is an extension which is unramified outside the dyadic primes, and let $\Gamma=\operatorname{Gal}(E / F)$. Then the transfer map induces the isomorphism

$$
\mathrm{K}_{2 n}\left(R_{E}\right)\{2\}_{\Gamma} \stackrel{\cong}{\longrightarrow} \mathrm{K}_{2 n}\left(R_{F}\right)\{2\} .
$$

Proof. The Tate spectral sequence (cf. [Se, Appendix 1]) specializes to a second quadrant cohomological spectral sequence

$$
E_{2}^{-p, q}=\mathrm{H}_{p}\left(\Gamma, \mathrm{H}_{\text {ét }}^{q}\left(R_{E} ; \mathbb{Q}_{2} / \mathbb{Z}_{2}(i)\right)\right) \Rightarrow \mathrm{H}_{\text {ét }}^{-p+q}\left(R_{F} ; \mathbb{Q}_{2} / \mathbb{Z}_{2}(i)\right) .
$$

The edge maps in this spectral sequence are the corestriction maps. Note that $E_{2}^{-p, q}$ is zero for $i, q \geq 2$ by [RW, Proposition 4.6]. Hence corestriction induces an isomorphism $\mathrm{H}_{\text {ét }}^{1}\left(R_{E} ; \mathbb{Q}_{2} / \mathbb{Z}_{2}(i)\right)_{\Gamma} \rightarrow \mathrm{H}_{\text {ét }}^{1}\left(R_{F} ; \mathbb{Q}_{2} / \mathbb{Z}_{2}(i)\right)$ for $i \geq 2$. Since the Bloch-Lichtenbaum spectral sequence is compatible with corestriction, we get from [RW, Theorem 6.3] the isomorphism $\mathrm{K}_{2 n+1}\left(R_{E} ; \mathbb{Q}_{2} / \mathbb{Z}_{2}\right)_{\Gamma} \rightarrow \mathrm{K}_{2 n+1}\left(R_{F} ; \mathbb{Q}_{2} / \mathbb{Z}_{2}\right)$ for $n \geq 1$. Next, compare the Bockstein exact sequences:

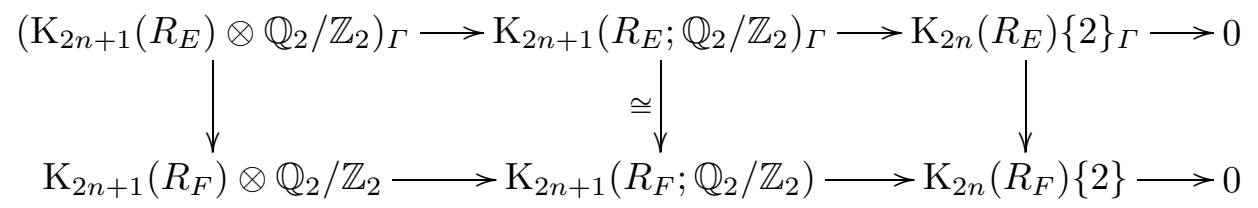

It follows immediately that $\mathrm{K}_{2 n}\left(R_{E}\right)\{2\}_{\Gamma} \rightarrow \mathrm{K}_{2 n}\left(R_{F}\right)\{2\}$ is surjective. Moreover, $\mathrm{K}_{2 n+1}\left(R_{F}\right) \otimes \mathbb{Q}_{2} / \mathbb{Z}_{2}$ injects into $\mathrm{K}_{2 n+1}\left(R_{F} ; \mathbb{Q}_{2} / \mathbb{Z}_{2}\right)$. The snake 
lemma implies that the kernel of $\mathrm{K}_{2 n}\left(R_{E}\right)\{2\}_{\Gamma} \rightarrow \mathrm{K}_{2 n}\left(R_{F}\right)\{2\}$ is divisible, hence trivial since $\mathrm{K}_{2 n}\left(R_{E}\right)$ is finite.

Denote by $\left(\bigoplus A_{i}\right)_{0}$ the subgroup of $\bigoplus A_{i}$ consisting of elements $a=\left(a_{i}\right)$ such that $\sum a_{i}=0$, in additive notation.

Proposition 6.4. If $\zeta_{2^{\nu}} \in E$ for some $\nu \geq 2$, then there is a natural short exact sequence of Galois modules

$$
0 \rightarrow \operatorname{Pic}\left(R_{E}\right) / 2^{\nu}(n) \rightarrow \mathrm{K}_{2 n}\left(R_{E}\right) / 2^{\nu} \rightarrow\left(\bigoplus_{S_{2}} \mathbb{Z} / 2^{\nu}\right)_{0}(n) \rightarrow 0 .
$$

Proof. The statement for $n=1$ is [Ta3, Theorem 6.2]. If $n>1$ we have

$$
\begin{aligned}
\mathrm{K}_{2 n}\left(R_{E}\right) / 2^{\nu} & =\mathrm{H}_{\text {ét }}^{2}\left(R_{E} ; \mathbb{Z}_{2}(n+1)\right) / 2^{\nu}=\mathrm{H}_{\text {ét }}^{2}\left(R_{E} ; \mathbb{Z} / 2^{\nu}(n+1)\right) \\
& =\mathrm{H}_{\text {ét }}^{2}\left(R_{E} ; \mathbb{Z} / 2^{\nu}(2)\right)(n-1)=\left(\mathrm{H}_{\text {ét }}^{2}\left(R_{E} ; \mathbb{Z}_{2}(2)\right) / 2^{\nu}\right)(n-1) \\
& =\left(\mathrm{K}_{2}\left(R_{E}\right) / 2^{\nu}\right)(n-1) .
\end{aligned}
$$

The group $\mathrm{H}_{\text {et }}^{3}\left(R_{E} ; \mathbb{Z}_{2}(n+1)\right)$ is trivial, so the second and fourth isomorphisms follow from the Bockstein exact sequence. The third isomorphism uses the fact that $\zeta_{2^{\nu}} \in E$. Theorem 6.14(a) of [RW] implies the remaining claims.

Following $[\mathrm{Ke}]$, we denote by $E^{\prime}$ the maximal Abelian unramified extension of $F\left(\zeta_{2^{\nu}}\right)$ for which $\Delta=\operatorname{Gal}\left(E^{\prime} / F\left(\zeta_{2^{\nu}}\right)\right)$ has exponent $2^{\nu}$ and the Archimedean and dyadic primes in $F\left(\zeta_{2^{\nu}}\right)$ split completely in $E^{\prime}$. That is, the Artin map in class field theory induces an isomorphism $\operatorname{Pic}_{+}\left(R_{F\left(\zeta_{2^{\nu}}\right)}\right) / 2^{\nu}$ $\rightarrow \Delta$ of $\Gamma$-modules. The field $E^{\prime}$ is the class field of $F\left(\zeta_{2^{\nu}}\right)$ in which the primes that represent the trivial element of $\operatorname{Pic}_{+}\left(R_{F\left(\zeta_{2} \nu\right.}\right) / 2^{\nu}$ split completely. Let $\Sigma$ denote the Galois group of $E^{\prime} / F$, and $T_{2}^{\prime}$ the set of dyadic primes in $E^{\prime}$.

Corollary 6.6. With the same assumption as in Proposition 6.4, the $\Sigma$-module map $\mathrm{K}_{2 n}\left(R_{E^{\prime}}\right) / 2^{\nu} \rightarrow\left(\bigoplus_{T_{2}^{\prime}} \mathbb{Z} / 2^{\nu}\right)_{0}(n)$ induces isomorphisms

of $\Gamma$-modules, and

$$
\mathrm{K}_{2 n}\left(R_{F\left(\zeta_{2}\right)}\right) / 2^{\nu} \stackrel{\cong}{\longrightarrow}\left(\left(\bigoplus_{T_{2}^{\prime}} \mathbb{Z} / 2^{\nu}\right)_{0}(n)\right)_{\Delta}
$$

$$
\mathrm{K}_{2 n}\left(R_{F}\right) / 2^{\nu} \stackrel{\cong}{\longrightarrow}\left(\left(\bigoplus_{T_{2}^{\prime}} \mathbb{Z} / 2^{\nu}\right)_{0}(n)\right)_{\Sigma} .
$$

Proof. Proposition 6.4 yields the diagram:




See Proposition 6.3 for the indicated $\Gamma$-module isomorphism. The left vertical map is induced by the norm map, hence is zero by the choice of $E^{\prime}$. It follows that the map $\left(\mathrm{K}_{2 n}\left(R_{E^{\prime}}\right) / 2^{\nu}\right)_{\Delta} \rightarrow\left(\left(\bigoplus_{T_{2}^{\prime}} \mathbb{Z} / 2^{\nu}\right)_{0}(n)\right)_{\Delta}$ is an isomorphism. This implies the $\Gamma$-module isomorphism. The remaining claim follows from Proposition 6.3.

Proof of Theorem 6.1 (after F. Keune). We will only sketch a proof; further details can be found in $[\mathrm{Ke},(7.4)-(7.6)]$ and [Hut]. By Corollary 6.6 and our assumptions, the long exact sequence in group homology associated to the short exact sequence $0 \rightarrow\left(\bigoplus_{T_{2}^{\prime}} \mathbb{Z} / 2^{\nu}\right)_{0}(n) \rightarrow \bigoplus_{T_{2}^{\prime}} \mathbb{Z} / 2^{\nu}(n) \rightarrow$ $\mathbb{Z} / 2^{\nu}(n) \rightarrow 0$ of $\Sigma$-modules reduces to the exact sequence

$$
\begin{aligned}
0 \rightarrow \mathrm{H}_{1}\left(\Sigma, \mathbb{Z} / 2^{\nu}(n)\right) \rightarrow \mathrm{K}_{2 n}\left(R_{F}\right)\{2\} & \\
& \rightarrow \bigoplus_{S_{2}} \mathrm{H}_{\text {ét }}^{2}\left(F_{\wp} ; \mathbb{Z}_{2}(n+1)\right) \rightarrow \mathrm{H}_{\text {ét }}^{0}\left(F ; \mathbb{Q}_{2} / \mathbb{Z}_{2}(n)\right) \rightarrow 0 .
\end{aligned}
$$

Moreover, $\mathrm{H}_{1}\left(\Sigma, \mathbb{Z} / 2^{\nu}(n)\right)=\mathrm{H}_{1}\left(\Delta, \mathbb{Z} / 2^{\nu}(n)\right)_{\Gamma}=\left(\Delta / 2^{\nu}(n)\right)_{\Gamma}$. Now compare (6.2) and (6.7).

7. Fitting ideals of wild kernels. Let $R$ be a unital commutative ring. If $M$ is a finitely generated $R$-module, let $\operatorname{Fit}_{R}(M)$ denote the first Fitting ideal of $M$ over $R$. See [MW] and [No] for an introduction to Fitting ideals. Let $\mathbb{Z}_{2}$ denote the 2 -adic integers.

Assume $F$ is an Abelian number field with conductor $f$. Let $\operatorname{Gal}(F / \mathbb{Q})=$ $P \times \Delta$ where $P$ is the 2-Sylow subgroup. We consider the 2-adic characters $\Delta \rightarrow \overline{\mathbb{Q}}_{2}^{\times}$up to $\operatorname{Gal}\left(\overline{\mathbb{Q}}_{2} / \mathbb{Q}_{2}\right)$-conjugacy. By adjoining the values of a character $\chi$ to $\mathbb{Z}_{2}$, we obtain a discrete valuation ring $\mathcal{O}_{\chi}$, and also a $\mathbb{Z}_{2}[\Delta]$-algebra via $\delta \cdot x:=\chi(\delta) x$ for $\delta \in \Delta, x \in \mathcal{O}_{\chi}$. If $M$ is a $\mathbb{Z}_{2}[\operatorname{Gal}(F / \mathbb{Q})]$-module, the $\chi$-eigenspace $M(\chi)=\mathcal{O}_{\chi} \otimes_{\mathbb{Z}_{2}[\Delta]} M$ is an $\mathcal{O}_{\chi}[P]$-module and there is a decomposition into eigenspaces

$$
M=\bigoplus_{[\chi]} M(\chi) .
$$

Recall the partial zeta-function $\zeta_{f}(s, a)=\sum_{k \equiv a \bmod f} k^{-s}$ where $a$ is an integer relatively prime to $f$, and $\operatorname{Re}(s)>1$. Choose a primitive $f$ th root of unity $\zeta_{f}$. For every positive $a$ with $(a, f)=1$, denote by $\sigma_{a} \in \operatorname{Gal}(F / \mathbb{Q})$ the restriction to $F$ of the automorphism of $\mathbb{Q}\left(\zeta_{f}\right)$ that maps $\zeta_{f}$ to its ath power. For $n \geq 0$, the $n$th Stickelberger element relative to $F$ is

$$
\theta_{n}=\sum_{(a, f)=1,1 \leq a<f} \zeta_{f}(-n, a) \sigma_{a}^{-1} .
$$

Let $D$ and $I$ denote the common decomposition and inertia groups of the dyadic primes in $F$. We can now state the main result of this section: 
TheOREM 7.1. Assume $F$ is a totally real Abelian number field of odd prime power conductor. Write $G=\operatorname{Gal}(F / \mathbb{Q})=\Delta \times P$, where $P$ is the 2-Sylow subgroup of $G$. Let $\chi: \Delta \rightarrow \overline{\mathbb{Q}}_{2}^{\times}$be a non-trivial 2-adic character. Assume $D \subset \Delta$, and let $\xi$ denote the restriction of $\chi$ to $D$. Put $a=4$ if $\xi$ is trivial and $a=2$ if $\xi$ is non-trivial. For $n \equiv 2 \bmod 4$ we have

$$
\mathrm{Fit}_{\mathbb{Z}_{2}[G](\chi)} \mathrm{WK}_{n}(F)\{2\}(\chi)=\left(\frac{\theta_{n / 2}(\chi)}{a}\right)
$$

where $\theta_{n / 2}$ is the $(n / 2)$ th Stickelberger element relative to $F$.

The Fitting ideals of the two-primary algebraic K-groups of $R_{F}$ were computed in [CØ, Corollary 1.3]. To compute the Fitting ideals of the wild kernels it remains to consider the other terms in the exact sequence in Corollary 4.1 .

It seems appropriate to make some comments on the assumptions in Theorem 7.1. For a dyadic prime $\wp$ there is an associated Galois extension $F_{\wp} / \mathbb{Q}_{2}$ with group $D$. Let $X\left(F_{\wp}, i\right)$ and $X(F, i)$ be short for $\mathrm{H}_{\text {ét }}^{0}\left(F_{\wp} ; \mathbb{Q}_{2} / \mathbb{Z}_{2}(i)\right)$ and $\bigoplus_{S_{2}} X\left(F_{\wp}, i\right)$. Note that $\bigoplus_{S_{2}} \mathrm{H}_{\text {ét }}^{2}\left(F_{\wp} ; \mathbb{Z}_{2}(i+1)\right)$ is isomorphic to $X(F, i)$ by Theorem 2.2. It is not true in general that $X(F, i)$ is $G$-cohomologically trivial. However, $X(F, i)$ is the induction $\operatorname{Ind}_{D}^{G}\left(X\left(F_{\wp}, i\right)\right)$ of $X\left(F_{\wp}, i\right)$ from $D$ to $G$ and there is the Galois descent isomorphism $X\left(F_{\wp}, i\right)^{I}=X\left(F_{\wp}^{\prime}, i\right)$ where $F_{\wp}^{\prime}$ is the unramified part of the extension $F_{\wp} / \mathbb{Q}_{2}$. So a necessary condition for $X(F, i)$ to be $G$-cohomologically trivial is that $X\left(F_{\wp}^{\prime}, i\right)$ is $D / I$-cohomologically trivial. Note that $X\left(F_{\wp}^{\prime}, i\right)=X\left(\mathbb{Q}_{2}, i\right)$, which is a nontrivial group for all $i$. Hence $X\left(F_{\wp}^{\prime}, i\right)$ is $D / I$-cohomologically trivial if and only if $D / I$ has odd order. From our assumptions we see that $I=0$ and $D$ has odd order. Hence $X\left(F_{\wp}, i\right)$ is $D$-cohomologically trivial, and it follows that $X(F, i)$ is $G$-cohomologically trivial being the induction of $X\left(F_{\wp}, i\right)$ from $D$ to $G$.

Proof. Let $n \equiv 2 \bmod 8$. The real primes of $F$ give a Fitting ideal equal to (2). Since $X(F, n / 2)$ is $G$-cohomologically trivial, [CG, Lemma 3] implies

$$
\begin{aligned}
\mathrm{Fit}_{\mathbb{Z}_{2}[G](\chi)} & \mathrm{K}_{n}(F)\{2\}(\chi) \\
= & (2) \cdot \operatorname{Fit}_{\mathbb{Z}_{2}[G](\chi)} \mathrm{WK}_{n}(F)\{2\}(\chi) \cdot \operatorname{Fit}_{\mathbb{Z}_{2}[G](\chi)} X(F, n / 2)(\chi) .
\end{aligned}
$$

Assume $\xi$ is the trivial character. From the parity of $n$, one finds the equalities

$$
\begin{aligned}
\mathrm{Fit}_{\mathbb{Z}_{2}[G](\chi)} X(F, n / 2)(\chi) & =\operatorname{Fit}_{\mathbb{Z}_{2}[P]} X\left(F_{\wp}, n / 2\right)(\xi) \\
& =\operatorname{Fit}_{\mathbb{Z}_{2}[P]} X\left(\mathbb{Q}_{2}, n / 2\right)=\left(w_{n / 2}^{(2)}\left(\mathbb{Q}_{2}\right)\right)=(2) .
\end{aligned}
$$

If $\xi$ is non-trivial, then $X\left(F_{\wp}, n / 2\right)(\xi)$ is the trivial group and hence $X(F, n / 2)(\chi)=0$. It remains to compute the Fitting ideal of $\mathrm{K}_{n}\left(R_{F}\right)\{2\}(\chi)$ over $\mathbb{Z}_{2}[G](\chi)$. This is done in [CØ, Corollary 1.3], and the result follows.

A similar computation implies the claim for $n \equiv 6 \bmod 8$. 
Theorem 7.1 implies structural results on the wild kernels. See [C $\varnothing]$ for the higher even K-groups. We mention two consequences:

COROLlary 7.2. Assume the hypothesis of Theorem 7.1. For all natural numbers $n \equiv 2 \bmod 4$ one has

$$
\# \mathrm{WK}_{n}(F)\{2\}(\chi) \sim_{2} \prod_{\chi^{\prime} \sim \chi} \prod_{\psi^{\prime} \mid \chi^{\prime}} \frac{1}{a} L\left(-n / 2, \psi^{\prime-1}\right) \sim_{2} \prod_{\chi^{\prime} \sim \chi} \prod_{\psi^{\prime} \mid \chi^{\prime}} \frac{B_{n / 2+1, \psi^{\prime}-1}}{a(n / 2+1)} .
$$

Here $\chi^{\prime} \sim \chi$ denotes that $\chi^{\prime}$ and $\chi$ are conjugate, and $\psi^{\prime} \mid \chi^{\prime}$ denotes that $\psi^{\prime}$ is a character of $G$ which extends $\chi^{\prime}$.

Proof. The order of $\mathrm{K}_{n}\left(R_{F}\right)\{2\}(\chi)$ is computed in [CØ, Corollary 3.14], and the formula above follows by the same method.

Corollary 7.3. Let $n \equiv 2 \bmod 4$ and assume the hypothesis of Theorem 7.1. In addition assume that $\mathrm{Pic}_{+}\left(\mathcal{O}_{\chi^{-1} \omega^{n / 2+1}}\right)\left(\chi^{-1} \omega^{n / 2+1}\right)$ is a cyclic $\mathcal{O}_{\chi^{-1} \omega^{n / 2+1}-m o d u l e}$ and that there is only one prime lying above 2 in $\mathbb{Q}_{\chi^{-1} \omega^{n / 2+1}}$. Then

$$
\mathrm{WK}_{n}(F)\{2\}(\chi)=\mathcal{O}_{\chi}[P] /\left(\frac{\theta_{n / 2}(\chi)}{a}\right) .
$$

Proof. This follows from [CØ, Corollary 4.4] and Theorem 7.1.

8. Leopoldt's conjecture and rank formulas. In this section we obtain an interpretation of the Leopoldt conjecture for totally imaginary number fields at the prime 2 in terms of wild kernels, and second we give higher 2-rank formulas for $\mathrm{WK}_{2 n}(F)$ in case $F$ is non-exceptional.

Fix a totally imaginary number field $F$. The group ker $\alpha / 2^{\nu}\left(R_{F}, 0\right)$ classifies the cyclic degree $2^{\nu}$ extensions of $F$ which are unramified outside the dyadic primes and locally embeddable in a $\mathbb{Z}_{2}$-extension (cf. [Iw] and [Ng1]). The proof of Proposition 4.8 implies the following two-primary version of a result in $[\mathrm{Ko} 3]$.

Proposition 8.1. Let $F$ be a totally imaginary number field and $m$ a natural number such that $m e_{\nu}>1$. Then the number of independent cyclic extensions of $F$ of degree $2^{\nu}$ which are unramified outside the dyadic primes and locally embeddable into a $\mathbb{Z}_{2}$-extension is equal to $\mathrm{rk}_{2}{ }^{\nu} \mathrm{WK}_{2 m e_{\nu}-2}(F)+$ $r_{2}(F)+1$.

Recall that the Leopoldt conjecture for $F$ at the prime 2 predicts that the number of independent $\mathbb{Z}_{2}$-extensions of $F$ is precisely $r_{2}(F)+1$.

Corollary 8.2. Assume the hypothesis of Proposition 8.1. If the rank $\mathrm{rk}_{2} \mathrm{WK}_{2 m e_{\nu}-2}(F)$ is zero, then the Leopoldt conjecture holds for $F$ at 2.

REMARK 8.3. If the 8-rank of $\mathrm{WK}_{2}(F)$ is zero, then the Leopoldt conjecture holds for $F$ at the prime 2. See also Lemma 4.6. 
Next we indicate how to prove higher 2-rank formulas for $\mathrm{WK}_{2 n-2}(F)$, provided $F$ is non-exceptional. The Lyndon-Hochschild-Serre spectral sequence

$$
E_{2}^{m, n}=\mathrm{H}^{m}\left(\Gamma^{\prime}, \mathrm{H}_{\text {ét }}^{n}\left(R_{F_{\infty}} ; \mathbb{Z} / 2^{\nu}(i)\right)\right) \Rightarrow \mathrm{H}_{\text {ét }}^{m+n}\left(R_{F} ; \mathbb{Z} / 2^{\nu}(i)\right)
$$

collapses at its $E_{2}$-page. In particular, there is the short exact sequence

$$
\begin{aligned}
0 \rightarrow \mathrm{H}^{1}\left(\Gamma^{\prime}, \mathbb{Z} / 2^{\nu}(i)\right) \rightarrow \mathrm{H}_{\text {êt }}^{1}\left(R_{F} ; \mathbb{Z} / 2^{\nu}(i)\right) & \\
& \rightarrow \mathrm{H}_{\text {ét }}^{1}\left(R_{F_{\infty}} ; \mathbb{Z} / 2^{\nu}(i)\right)^{\Gamma^{\prime}} \rightarrow 0 .
\end{aligned}
$$

Let $\overline{\operatorname{ker}} \alpha / 2^{\nu}\left(R_{F}, i\right)$ denote the quotient of $\operatorname{ker} \alpha / 2^{\nu}\left(R_{F}, i\right)$ obtained by dividing out by $\mathrm{H}^{1}\left(\Gamma^{\prime}, \mathbb{Z} / 2^{\nu}(i)\right)$ under the injection in (8.4). The Galois descent isomorphism

$$
\overline{\operatorname{ker}} \alpha / 2^{\nu}\left(R_{F_{k}}, i\right)^{\Gamma_{k}}=\overline{\operatorname{ker}} \alpha / 2^{\nu}\left(R_{F}, i\right)
$$

follows as in Lemma 5.8. Let $2^{e}$ denote the order of the group of two-primary roots of unity in $F$, and let $\nu=e+k$. If $i$ is even, there is the exact sequence

$$
\begin{aligned}
0 \rightarrow\left(\operatorname{ker} g_{k} / \mu_{2^{\nu}} \otimes \mathbb{Z} / 2^{\nu}(i-1)\right)^{\Gamma_{k}} & \rightarrow \overline{\operatorname{ker}} \alpha / 2^{\nu}\left(R_{F}, i\right) \\
& \rightarrow{ }^{\nu} J_{F_{k}} / \overline{\mathbf{F}_{k} X_{F_{k}}}(i-1)^{\Gamma_{k}} \rightarrow 0,
\end{aligned}
$$

which is the finite version of the short exact sequence in Theorem 5.16. Exactness of (8.5) follows since ker $g_{k} / \mu_{2^{\nu}} \otimes \mathbb{Z} / 2^{\nu}(i-1)$ is a cohomologically trivial $\Gamma_{k}$-module (cf. [Me, Lemma 2]). This uses the fact that ker $g_{k} / \mu_{2^{\nu}}$ is two-torsion-free by the choice of $\nu$, and that $\Gamma_{k}$ is cyclic by the assumption on $F$.

TheOREM 8.6. Let $F$ be a non-exceptional number field. Let $\nu=e+k$ with $e$ as above, and let $n \geq 2$ be even. Then

$\operatorname{rk}_{2^{\nu}} \mathrm{WK}_{2 n-2}(F)=\operatorname{rk}_{2^{\nu}}\left(\operatorname{ker} g_{k} / \mu_{2^{\nu}} \otimes \mathbb{Z} / 2^{\nu}(n-1)\right)^{\Gamma_{k}}$

$$
+\operatorname{rk}_{2}\left(2^{\nu} J_{F_{k}} / \overline{\mathbf{F}_{k} X_{F_{k}}}(n-1)^{\Gamma_{k}} / \operatorname{im} \gamma_{n}^{\nu}\right)-r_{2}(F),
$$

where $\gamma_{n}^{\nu}:{ }_{2}{ }^{\nu-1} J_{F_{k-1}} / \overline{\mathbf{F}_{k-1} X_{F_{k-1}}}(n-1)^{\Gamma_{k-1}} \rightarrow{ }_{2^{\nu}} J_{F_{k}} / \overline{\mathbf{F}_{k} X_{F_{k}}}(n-1)^{\Gamma_{k}}$.

Proof. Let $\bar{K}_{2 n-1}\left(R_{F}\right)$ denote $\mathrm{K}_{2 n-1}\left(R_{F}\right)$ modulo its two-torsion subgroup. By the exact sequence

$$
0 \rightarrow \bar{K}_{2 n-1}\left(R_{F}\right) / 2^{\nu} \rightarrow \overline{\operatorname{ker}} \alpha / 2^{\nu}\left(R_{F}, n\right) \rightarrow{ }_{2}{ } \mathrm{WK}_{2 n-2}(F) \rightarrow 0,
$$

it suffices to compute the 2 -rank of $\overline{\operatorname{ker}} \alpha / 2^{\nu}\left(R_{F}, n\right)$. Note that (8.7) is split since $\bar{K}_{2 n-1}\left(R_{F}\right) / 2^{\nu}=\left(\mathbb{Z} / 2^{\nu}\right)^{r_{2}(F)}$ is a pure subgroup. We conclude using (8.5) and the arguments of [Ko2, p. 155]. Details will be left to the reader.

9. Divisible elements in algebraic K-groups. Let $A$ be a (multiplicative) Abelian group. Its subgroup of divisible elements $\operatorname{div} A$ is $\bigcap_{n \geq 1} A^{n}$. 
Let $p$-div $A$ denote the subgroup of $p$-divisible elements. The maximal divisible subgroup Div $A$ of $A$ is contained in $\operatorname{div} A$. Let $\operatorname{div} A /$ Div denote the quotient.

Lemma 9.1. There is an isomorphism $\operatorname{div} A / \operatorname{Div}=\operatorname{div}(A / \operatorname{Div} A)$.

Proof. Write $A=B \oplus \operatorname{Div} A$ where $B$ has no non-zero divisible subgroup, and note that both the groups in question are isomorphic to div $B$.

For $n \geq 1$, Quillen's Theorem $5([\mathrm{Qu} 2, \S 5])$ specializes to short exact sequences

$$
0 \rightarrow \mathrm{K}_{2 n}\left(R_{F}\right) \rightarrow \mathrm{K}_{2 n}(F) \rightarrow \bigoplus_{\wp \notin S_{p}} \mathrm{~K}_{2 n-1}(F[\wp]) \rightarrow 0 .
$$

(See [Sou, Théorème 3] and [We, Theorem 4.6].) It follows that $\mathrm{K}_{2 n}(F)$ is an extension of a sum of finite groups by a finite group (cf. [Bo, Proposition 12.2], [Qu1, Theorem 8(i)] and [Qu3, Theorem 1]). Hence $\mathrm{K}_{2 n}(F)$ is a torsion group with no non-trivial divisible subgroups, i.e., reduced, and $p$-div $\mathrm{K}_{2 n}(F)$ is a subgroup of $\mathrm{K}_{2 n}\left(R_{F}\right)\{p\}$. See [Ba1] and [Ba2] for examples where $p$-div $\mathrm{K}_{2 n}(F)$ is non-trivial. By [Ba2, Theorem 3(i)], $\mathrm{WK}_{2 n}^{\text {ét }}(F)\{p\}$ is isomorphic to $p$-div $\mathrm{K}_{2 n}(F)$ if $p$ is odd. Below we discuss this isomorphism for the wild kernel when $p=2$ and $F$ is non-exceptional.

Recall from [Ja] the continuous $p$-adic étale cohomology groups $\mathrm{H}_{\text {ét }}^{n}(F$; $\left.\mathbb{Z}_{2}(i)\right)$. By [Ja, (0.2)] there is the Milnor $\lim ^{1}$ exact sequence

$$
0 \rightarrow \lim _{\nu}{ }^{1} \mathrm{H}_{\text {ét }}^{n-1}\left(F ; \mathbb{Z} / 2^{\nu}(i)\right) \rightarrow \mathrm{H}_{\text {ét }}^{n}\left(F ; \mathbb{Z}_{2}(i)\right) \rightarrow \lim _{\nu} \mathrm{H}_{\text {ét }}^{n}\left(F ; \mathbb{Z} / 2^{\nu}(i)\right) \rightarrow 0 .
$$

LEMMA 9.2. There is an isomorphism

$$
\operatorname{div} \mathrm{H}_{\text {ét }}^{2}\left(F ; \mathbb{Z}_{p}(n)\right)=\lim _{\nu}{ }^{1} \mathrm{H}_{\text {ét }}^{1}\left(F ; \mathbb{Z} / p^{\nu}(n)\right) .
$$

Proof. Note that

$$
\operatorname{div} \mathrm{H}_{\text {ét }}^{2}\left(F ; \mathbb{Z}_{p}(n)\right)=\operatorname{ker}\left(\mathrm{H}_{\text {ét }}^{2}\left(F ; \mathbb{Z}_{p}(n)\right) \rightarrow \lim _{\nu} \mathrm{H}_{\text {ét }}^{2}\left(F ; \mathbb{Z}_{p}(n)\right) / p^{\nu}\right) .
$$

Via the Milnor $\lim ^{1}$ exact sequence, it suffices to identify the limits $\lim _{\nu} \mathrm{H}_{\text {ét }}^{2}\left(F ; \mathbb{Z}_{p}(n)\right) / p^{\nu}$ and $\lim _{\nu} \mathrm{H}_{\text {ét }}^{2}\left(F ; \mathbb{Z} / p^{\nu}(n)\right)$. This follows immediately from the Bockstein exact sequence

$$
0 \rightarrow \mathrm{H}_{\text {ét }}^{2}\left(F ; \mathbb{Z}_{p}(n)\right) / p^{\nu} \rightarrow \mathrm{H}_{\text {ét }}^{2}\left(F ; \mathbb{Z} / p^{\nu}(n)\right) \rightarrow p^{\nu} \mathrm{H}_{\text {ét }}^{3}\left(F ; \mathbb{Z}_{p}(n)\right) \rightarrow 0
$$

and [Ta1, Theorem 3.1(c)].

Concerning the maximal $p$-torsion subgroup of $\mathrm{H}_{\text {ét }}^{n}\left(F ; \mathbb{Z}_{p}(i)\right)$ we have:

Proposition 9.3. For $i \geq 2$ there is a short exact sequence

$$
0 \rightarrow \mathrm{H}_{\text {ét }}^{2}\left(R_{F} ; \mathbb{Z}_{p}(i)\right) \rightarrow \mathrm{H}_{\text {ét }}^{2}\left(F ; \mathbb{Z}_{p}(i)\right)\{p\} \rightarrow \bigoplus_{\wp \notin S_{p}} \mathrm{H}_{\text {ét }}^{1}\left(F[\wp] ; \mathbb{Z}_{p}(i-1)\right) \rightarrow 0 .
$$

In particular, $\mathrm{H}_{\text {ét }}^{2}\left(F ; \mathbb{Z}_{p}(i)\right)\{p\}$ is a torsion group with no non-zero divisible subgroup. 
Proof. Since $\lim _{\nu}^{1} \mathrm{H}_{\mathrm{e} t}^{0}\left(F ; \mathbb{Z} / p^{\nu}(i)\right)=0$ and likewise for $R_{F}$ and all the residue fields, [Sou, Proposition 1] implies the exact sequence

$$
0 \rightarrow \mathrm{H}_{\text {ét }}^{1}\left(R_{F} ; \mathbb{Z}_{p}(i)\right) \rightarrow \mathrm{H}_{\text {ét }}^{1}\left(F ; \mathbb{Z}_{p}(i)\right) \rightarrow \bigoplus_{\wp \notin S_{p}} \mathrm{H}_{\text {ét }}^{0}\left(F[\wp] ; \mathbb{Z}_{p}(i-1)\right) .
$$

Here $\mathrm{H}_{\text {ét }}^{0}\left(F[\wp] ; \mathbb{Z}_{p}(i-1)\right)=0$ for $i \neq 1$ by [RW, Propositions 1.10 and 2.4(b)]. Let $W$ denote $\mathbb{Q}_{p} / \mathbb{Z}_{p}$ and consider the diagram of Bockstein exact sequences:

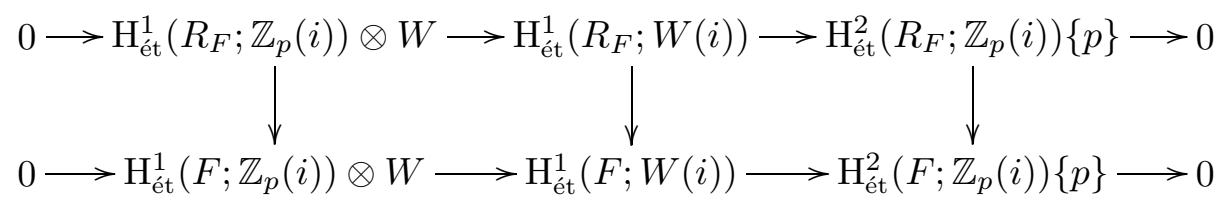

The left vertical map is an isomorphism from (9.4). By [RW, Proposition 4.7], there is for $i \geq 1$ the exact sequence

$$
0 \rightarrow \mathrm{H}_{\text {ét }}^{1}\left(R_{F} ; W(i)\right) \rightarrow \mathrm{H}_{\text {ét }}^{1}(F ; W(i)) \rightarrow \bigoplus_{\wp \notin S_{p}} \mathrm{H}_{\text {ét }}^{0}(F[\wp] ; W(i-1)) \rightarrow 0 .
$$

This proves the injectivity statement in the proposition. By [RW, Propositions 1.10 and $2.4(\mathrm{~b})]$, we may identify $\mathrm{H}_{\mathrm{ét}}^{0}(F[\wp] ; W(i-1))$ and $\mathrm{H}_{\text {ét }}^{1}\left(F[\wp] ; \mathbb{Z}_{p}(i-1)\right)$. To conclude we use that $\mathrm{H}_{\text {ét }}^{2}\left(R_{F} ; \mathbb{Z}_{p}(i)\right)$ is finite for $i \geq 2$. See [RW, Proposition 6.12] if $p=2$, and [DF, Theorem 8.7, Remark 8.8] if $p$ is odd.

Let $p=2$. The proof of Theorem 9.5 uses a comparison with étale cohomology, namely $\mathrm{K}_{2 n}(F)\{2\}=\mathrm{H}_{\text {ét }}^{2}\left(F ; \mathbb{Z}_{2}(n+1)\right)\{2\}$ for $n \geq 1$ and $F$ totally imaginary. Let $i=n+1$ in the Bockstein exact sequences:

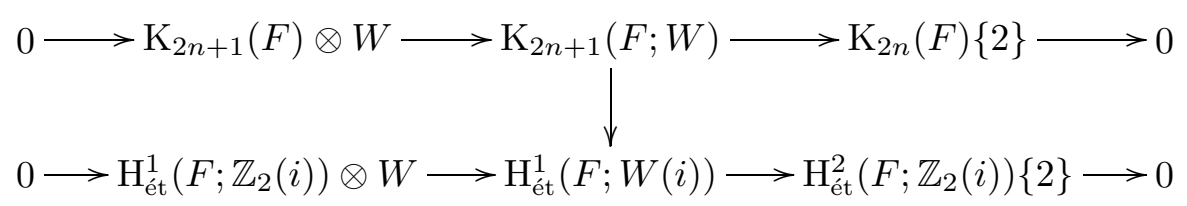

The vertical map is the edge map in the Bloch-Lichtenbaum spectral sequence for $F$, which is bijective by [RW, Theorem 1.13]. The image of $\mathrm{K}_{2 n+1}(F) \otimes W$ under the zig-zag map is trivial by Proposition 9.3. It follows easily from the above that the induced right hand vertical map is an isomorphism (cf. Proposition 6.3).

Theorem 9.5. Let $F$ be a non-exceptional number field and $n \geq 1$. Then

$$
2 \text {-div } \mathrm{K}_{2 n}(F)=\mathrm{WK}_{2 n}(F)\{2\} .
$$

Proof. We have the following commutative diagram (cf. [Ta3, Proposition 2.3]): 


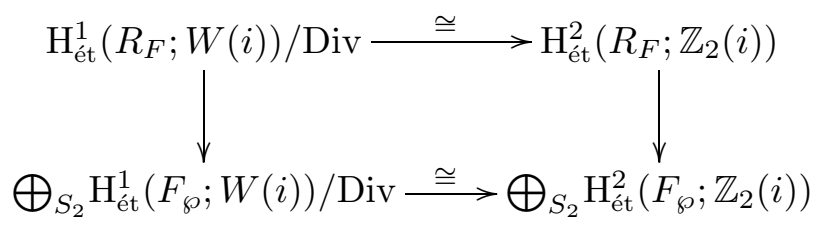

By the discussion above, it suffices to prove that $\operatorname{div} \mathrm{H}_{\text {ét }}^{2}\left(F ; \mathbb{Z}_{2}(i)\right)\{2\}$ is the kernel of the vertical map for $i=n+1$. Our proof follows [Sc, Lemma 4 in $\S 4$ ]. From the coefficient extension $0 \rightarrow \mathbb{Z} / 2^{\nu}(i) \rightarrow W(i) \rightarrow W(i) \rightarrow 0$ we find the commutative diagram where the product runs over all the finite primes of $F$ :



The right vertical map is injective by [Ne, Satz 4.5]. Here we use the assumption that $F$ is non-exceptional. Hence the left vertical map is injective, and moreover

$$
\begin{aligned}
\operatorname{div} & \mathrm{H}_{\text {ét }}^{1}(F ; W(i)) \\
& =\left\{x \in \mathrm{H}_{\text {ét }}^{1}(F ; W(i)) \mid \lambda_{\wp}(x) \in \operatorname{div} \mathrm{H}_{\text {ét }}^{1}\left(F_{\wp} ; W(i)\right) \text { for all } \wp\right\} \\
& \stackrel{(1)}{=}\left\{x \in \mathrm{H}_{\text {ét }}^{1}(F ; W(i)) \mid \lambda_{\wp}(x) \in \operatorname{Div~H}_{\text {ét }}^{1}\left(F_{\wp} ; W(i)\right) \text { for all } \wp\right\} \\
& \stackrel{(2)}{=}\left\{x \in \mathrm{H}_{\text {êt }}^{1}\left(R_{F} ; W(i)\right) \mid \lambda_{\wp}(x) \in \operatorname{Div~}_{\text {êt }}^{1}\left(F_{\wp} ; W(i)\right) \text { for } \wp \in S_{2}\right\} .
\end{aligned}
$$

Part (1) uses the fact that $\mathrm{H}_{\text {ét }}^{1}\left(F_{\wp} ; W(i)\right) /$ Div is cyclic of finite order. If $\wp$ is not a dyadic prime, then Div $\mathrm{H}_{\mathrm{ett}}^{1}\left(F_{\wp} ; W(i)\right)$ is the trivial group since $\mathrm{H}_{\text {ét }}^{1}\left(F_{\wp} ; W(i)\right)$ is a finite cyclic group. The local characterization of $\mathrm{H}_{\text {ét }}^{1}\left(R_{F} ; W(i)\right)$ in [Sc, Lemma 3ii) §4] implies (2). This finishes the proof since

$$
\operatorname{div} \mathrm{H}_{\text {ét }}^{1}(F ; W(i)) / \operatorname{Div}=\operatorname{div}\left(\mathrm{H}_{\text {ét }}^{1}(F ; W(i)) / \operatorname{Div}\right)=\operatorname{div} \mathrm{H}_{\text {ét }}^{2}\left(F ; \mathbb{Z}_{2}(i)\right)\{2\},
$$
by Lemma 9.1 and [Ta3, Proposition 2.3].

REMARK 9.6. Theorem 9.5 is not true in general for exceptional fields and $n=1$. This is discussed in [Hut].

\section{References}

[Ba1] G. Banaszak, Algebraic K-theory of number fields and rings of integers and the Stickelberger ideal, Ann. of Math. 135 (1992), 325-360.

[Ba2] - Generalization of the Moore exact sequence and the wild kernel for higher K-groups, Compositio Math. 86 (1993), 281-305. 
[BG1] G. Banaszak and W. Gajda, Euler systems for higher K-theory of number fields, J. Number Theory 58 (1996), 213-252.

[BG2] -, 一, On the arithmetic of cyclotomic fields and the K-theory of $\mathbb{Q}$, in: Algebraic K-theory (Poznań, 1995), G. Banaszak, W. Gajda and P. Krasoń (eds.), Contemp. Math. 199, Amer. Math. Soc., Providence, RI, 1996, 7-18.

[B-Z] G. Banaszak, W. Gajda, P. Krason and P. Zelewski, A note on the QuillenLichtenbaum conjecture and the arithmetic of square rings, K-Theory 16 (1999), 229-243.

[BP] F. Bertrandias et J.-J. Payan, $\Gamma$-extensions et invariants cyclotomiques, Ann. Sci. École Norm. Sup. (4) 5 (1972), 517-543.

[Bo] A. Borel, Stable real cohomology of arithmetic groups, ibid. 7 (1974), 235-272.

[CW] S. U. Chase and W. C. Waterhouse, Moore's theorem on uniqueness of reciprocity laws, Invent. Math. 16 (1972), 267-270.

[C-S] T. Chinburg, M. Kolster, G. Pappas and V. Snaith, Galois structure of K-groups of rings of integers, K-Theory 14 (1998), 319-369.

[CL] J. Coates and S. Lichtenbaum, On l-adic zeta-functions, Ann. of Math. 98 (1973), 498-550.

[CS] J. Coates and W. Sinnott, An analogue of Stickelberger's theorem for the higher K-groups, Invent. Math. 24 (1974), 149-161.

[CG] P. Cornacchia and C. Greither, Fitting ideals of class groups of real fields with prime power conductor, J. Number Theory 73 (1998), 459-471.

[CØ] P. Cornacchia and P. A. Østvær, On the Coates-Sinnott conjecture, K-Theory 19 (2000), 195-209.

[DF] W. G. Dwyer and E. M. Friedlander, Algebraic and étale K-theory, Trans. Amer. Math. Soc. 292 (1985), 247-280.

[Gi] R. Gillard, Formulations de la conjecture de Leopoldt et étude d'une condition suffisante, Abh. Math. Sem. Univ. Hamburg 48 (1979), 125-138.

[GJ] G. Gras et J.-F. Jaulent, Sur le corps de nombres réguliers, Math. Z. 202 (1989), 343-365.

[Gr] B. H. Gross, $p$-adic L-series at $s=0$, J. Fac. Sci. Univ. Tokyo Sect. IA Math. 28 (1981), 979-994.

[HM] L. Hesselholt and I. Madsen, On the K-theory of local fields, University of Aarhus preprint series 18 (1999).

[Hu] J. Hurrelbrink, On the wild kernel, Arch. Math. (Basel) 40 (1983), 316-318.

[Hut] K. Hutchinson, The 2-Sylow subgroup of the wild kernel of exceptional number fields, J. Number Theory 87 (2001), 222-238.

[Iw] K. Iwasawa, On $\mathbb{Z}_{l}$-extensions of algebraic number fields, Ann. of Math. 98 (1973), 246-326.

[Ja] U. Jannsen, Continuous étale cohomology, Math. Ann. 280 (1988), 207-245.

[Jau] J.-F. Jaulent, L'arithmétique des l-extensions (Thèse), Publ. Math. Fac. Sci. Besançon Théor. Nombres Années 1984/85-1985/86 1 (1986), 1-349.

[Ke] F. Keune, On the structure of the $\mathrm{K}_{2}$ of the ring of integers in a number field, K-Theory 2 (1989), 625-645.

[Ko1] M. Kolster, An idélic approach to the wild kernel, Invent. Math. 103 (1991), 9-24.

[Ko2] - , $\mathrm{K}_{2}$ of rings of algebraic integers, J. Number Theory 42 (1992), 103-122.

[Ko3] -, Remarks on étale K-theory and the Leopoldt's conjecture (Séminaire de Théorie des Nombres, Paris, 1991-1992), Progress in Math. 116, Birkhäuser, 1993, $37-62$.

[KM] M. Kolster and A. Movahhedi, Galois co-descent for étale wild kernels and capitulation, Ann. Inst. Fourier (Grenoble) 50 (2000), 35-65. 
$[\mathrm{MW}] \quad$ B. Mazur and A. Wiles, Class fields of Abelian extensions of $\mathbb{Q}$, Invent. Math. 76 (1984), 179-330.

[Me] A. S. Merkur'ev, Torsion in $\mathrm{K}_{2}$ groups of fields, Vestnik Leningrad Univ. Math. 21 (1988), 21-24.

[Mi] J. S. Milne, Arithmetic Duality Theorems, Perspect. Math. 1, Academic Press, 1986.

[Mo] C. Moore, Group extension of p-adic and adelic linear groups, Inst. Hautes Études Sci. Publ. Math. 35 (1969), 5-74.

[Ne] J. Neukirch, Über das Einbettungsproblem der algebraischen Zahlentheorie, Invent. Math. 21 (1973), 59-116.

[Ng1] T. Nguyen Quang Do, Sur la $\mathbb{Z}_{p}$-torsion de certains modules Galoisiens, Ann. Inst. Fourier (Grenoble) 36 (1986), 27-46.

[Ng2] - , Analogues supérieurs du noyau sauvage, J. Théor. Nombres Bordeaux 4 (1992), 263-271.

[No] D. G. Northcott, Finite Free Resolutions, Cambridge Tracts in Math. 71, Cambridge Univ. Press, 1976.

[Qu1] D. G. Quillen, On the cohomology and K-theory of the general linear group over a finite field, Ann. of Math. 96 (1972), 552-586.

[Qu2] -, Higher algebraic K-theory, I, in: Algebraic K-theory I, H. Bass (ed.), Lecture Notes in Math. 341, Springer, 1973, 85-147.

[Qu3] - Finite generation of the groups $\mathrm{K}_{i}$ of rings of algebraic integers, ibid., 179-198.

[RØ] J. Rognes and P. A. Østvær, Two-primary algebraic K-theory of two-regular number fields, Math. Z. 233 (2000), 251-263.

[RW] J. Rognes and C. A. Weibel, Two-primary algebraic K-theory of rings of integers in number fields, J. Amer. Math. Soc. 13 (2000), 1-54.

[Sc] P. Schneider, Über gewisse Galoiscohomologiegruppen, Math. Z. 168 (1979), 181-205.

[Se] J.-P. Serre, Cohomologie Galoisienne, 5th ed., Lecture Notes in Math. 5, Springer, 1994.

[Sou] C. Soulé, K-théorie des anneaux d'entiers de corps de nombres et cohomologie étale, Invent. Math. 55 (1979), 251-295.

[Ta1] J. Tate, Duality theorems in Galois cohomology over number fields, in: Proc. Intern. Congress Math., Stockholm, 1962, 234-241.

[Ta2] - Letter from Tate to Iwasawa on a relation between $\mathrm{K}_{2}$ and Galois cohomology, in: Algebraic K-theory II, H. Bass (ed.), Lecture Notes in Math. 342, Springer, $1973,524-527$.

[Ta3] -, Relations between $\mathrm{K}_{2}$ and Galois cohomology, Invent. Math. 36 (1976), $257-274$.

[We] C. A. Weibel, Étale Chern classes at the prime 2, in: Algebraic K-theory and Algebraic Topology, P. Goerss and J. F. Jardine (eds.), NATO Adv. Sci. Inst. Ser. C 407, Kluwer, 1993, 249-286.

Department of Mathematics

University of Oslo

Oslo, Norway

E-mail: paularne@math.uio.no 